\title{
Creative Visualisation in Chemistry
}

\author{
Jonathan P. Bowen* \\ Department of Informatics, School of Engineering \\ London South Bank University, \\ 103 Borough Road, London SE1 0AA, UK \\ E-mail: jonathan.bowen@1sbu.ac.uk \\ *Corresponding author
}

\author{
Alice M. Bowen \\ Institute for Physical and Theoretical Chemistry, \\ Goethe University Frankfurt am Main, \\ Max-von-Laue-Straße 7, D-60438 Frankfurt/Main, Germany \\ E-mail: bowen@prisner.de
}

\author{
Karl Harrison \\ Department of Chemistry, \\ University of Oxford, \\ South Parks Road, Oxford OX1 3QZ, UK \\ E-mail: karl.harrison@chem.ox.ac.uk
}

\begin{abstract}
Visualisation of molecules in the field of chemistry has been important for understanding their structure, whether simple or complicated. Initially this was done by hand, but latterly software has come to the aid of researchers and the vast majority of chemistry visualisation is now computer-generated. As well as aiding understanding, many molecules, especially if complex in nature, can take on an artistic quality when visualised, using artificial colour for example. Often these are used for creative reasons on the front of chemistry journals, for example, and sometimes as an inspiration for more pure art forms. This paper introduces molecular graphics in the context of creative computing. It also provides a history of the development of visualisation in chemistry, especially more recently with the use of software and the increasing use on journal covers. A brief survey of some of the software involved is included. Finally some conclusions are drawn with respect to the creative directions being taken now and possible directions in the future.
\end{abstract}

Keywords: 3D projection; digital art; electronic visualisation; molecular structure; virtual chemistry.

Biographical notes: Jonathan Bowen FBCS FRSA is Professor of Computer Science at Birmingham City University, Emeritus Professor Computing at London South Bank University, and Chairman of Museophile Limited. He has previously held academic posts at Imperial College London, University of Oxford, University of Reading, and London South Bank University. He has worked in industry including, for example, at Silicon Graphics and Altran Praxis. Bowen has held recent Visiting Professorship positions at the Pratt Institute (New York, USA), University of Waikato (New Zealand), and King's College London. His research interests include software engineering, electronic visualisation, and museum informatics. Since 2006, he has edited the proceedings of the EVA London Conference on Electronic Visualisation and the Arts. He is a Fellow of the British Computer Society, a Fellow of the Royal Society of Arts, and a Liveryman in the Worshipful Computer of Information Technologists.

Alice Bowen was awarded a D.Phil. doctorate in chemistry by the University of Oxford in 2013. She is currently a postdoctorial Research Associate and GOIN Fellow at Goethe University, Frankfurt am Main working within the research group of Prof. Thomas Prisner, in the area of Electron Paramagnetic Resonance (EPR) spectroscopy. Bowen's research uses a variety of EPR techniques to study the structure of proteins and model chemical systems. Bowen won the JEOL student lecture prize at the Royal Society of Chemistry ESR Group Conference in Manchester, UK, in 2012. She was also one of the 280 invited participants at the Global Young Scientists Summit 2013 held in Singapore and an invited speaker at the inaugural AWEST conference in Japan during 2013. 
Karl Harrison coordinates the Information Technology support service in the Department of Chemistry at the University of Oxford. He also provides undergraduate and graduate IT skills training and undertakes research to enhance the teaching and learning of chemistry supported by IT. His research investigations focus on the development of teaching software packages to enhance the learning of chemistry. Harrison has collaborated with Oxford University Press and has provided interactive resource materials for their chemistry text books. He has a strong research interest in science visualisations, interactive multimedia, metaverses, 3D environments, stereo projections, and panoramic photography. He has produced an online virtual tour of the city of Oxford.

\section{Background}

The history of human progress with respect to developing knowledge has depended on creativity and invention through the centuries (van Doren, 1991). Approaches have varied depending on the cultural environment. For example, linear thinking is favoured in Western cultures and a more holistic view has been prevalent historically in Eastern cultures (Alcorn, 1997). The necessary creativeness associated with science and art is a matter for debate, with scientific advances often being termed as discoveries rather than creations (Robinson, 2010a). Despite this, a creative nature is needed for significant advances in all disciplines. For course, different personalities can affect the way that the creative process is approached (Robinson, 2010b; Weisberg, 2006), but this variety is itself probably an important aspect of creativity. Sometimes a combination of different creative minds can be beneficial in achieving a significant advance.

Visualisation has been an important part of scientific progress, especially since the advent of photography. Often these visual techniques reveal beyond what can be detected with the human eye alone (Darius, 1984).With the availability of computers, it is possible to visualise in an even more abstract and complex way. For example, Stephen Wolfram explored the visualisation of cellular automata in his book A New Kind of Science (Wolfram, 2002). He also led the development of the Mathematica tool that allows mathematics to be captured and manipulated on a computer, providing visualisation facilities as well. Generation of these mathematical forms as graphical output can produce beautiful and artistic results (Bakshee, 1999; Trott, 1999). In general, much mathematics with its own intrinsic abstract beauty can also be visualised in interesting ways too (Bowen \& Giannini, 2015).

Computers have enabled digital art to be created. Initially computers were too expensive to be used for artistic purposes by all but a few pioneers with privileged access (Brown et al., 2008), but this has changed radically as costs have dropped dramatically. Now, computers together with the Internet have become ubiquitous and relatively cheap to use by any artist that wishes to do so (Greene, 2004). Mainstream artists such as David Hockney have embraced digital technology to produce completely digitally generated artworks, e.g., using the iPad (Gayford, 2011), as exhibited at the Royal Academy in London in his 'A Bigger Picture' exhibition of 2012. Grayson Perry has gone even further and has featured digital aspects of life, for example as a series of digitally woven tapestries presenting a story of the rise and fall of a computer scientist in a manner reminiscent of Hogarth (Perry, 2013).

Creativity can be seen as a process (Edmonds \& Quantrill, 1999). Sometimes the process is as important as the result and can be interdisciplinary, involving both arts and science (Bowen \& d'Inverno, 2006). With the almost ubiquitous availability of computing devices nowadays, creativity and computing often become intermixed. Creative computing may be said to be the use of computation in a novel, elegant, and perhaps artistic way, whereas computational creativity may be seen as using computers to achieve a creative result (Hugill \& Yang, 2013). The former is more about the process whereas the latter is more about the result. Both are important and worthwhile; indeed, they may be achieved together. For example, in the case of David Hockey, his process of using an iPad to produce art is of interest as well as the result of that process, the artwork itself. Sometimes it is possible to take this a step further and involve the end-user in the process as part of an interactive artwork, from example enabling remote communication on the Internet (Edmonds \& Franco, 2013).

Electronic visualisation is now an accepted part of the arts and culture in general (Bowen et al., 2013). The visualisation may be an aid to gaining an understanding of cognitive, emotional, social, ethical, or political aspects of an issue (Sula, 2013). This can potentially extend across any field of arts, humanities, or science, limited only by human creativity. In particular, for example, online communities and the relationships between participants can now be visualised relatively easily in an automated manner (Bowen, 2013b). 
Within this wider context of creativity and the use of IT, in this paper we consider the use of artistic visualisation within the academic field of chemistry (Hoffman, 2003; Laszlo, 2003), especially with relation to the viewing of molecular structures (Atkins, 2003). This is important both for the scientific understanding of the three-dimension nature of molecules and also for communicating the beauty of these structures to fellow scientists and the general public, perhaps for educational purposes (Jones, 2005). Promulgating the public understanding of science through the use of IT is increasingly important, especially online (Bowen \& Borda, 2009). The paper provides a historical view of developments in molecular visualisation, before and after the widespread availability of IT support, together with a survey of the current state of the art in terms of the images that are possible and the specialist software that is now available. A specific case study on the use in the context of chemistry journal covers is included.

In Section 2, we present some introductory context to the visualisation of molecules and how this has developed dramatically from the time before computers were used to the improved capabilities that IT can provide, using chlorophyll $a$ as a specific example. These developments have led to the widespread use of computer-generated visualisation with creative aspirations on the covers of chemistry journals. In Section 3, we give a more comprehensive historical account of the developments. In Section 4, we present modern visualisation examples of chemical modules, many with artistic qualities even if this is often not the main purpose. In Section 5, we give an example of the development of the artwork for the cover of a chemistry journal. Section 6 provides an overview of the software that is available for molecular visualisation. Finally in Section 7 we conclude that the visualisation of molecules is being increasingly used for both scientific and artistic reasons, with further developments in more dynamic visualisation likely in the future.

\section{Context}

Chemists have had a need to use visualisation to advance their science, ever before the use of computers, especially with regard to molecules of any complexity. The three-dimension nature of molecules means that it is important to understand the spatial relationship between atoms. A highly important example is the discovery of the structure of DNA by James Crick and Francis Watson at the University of Cambridge in 1953. They used models to explore the structure, leading to the understanding of the double helix nature of DNA (Figure 1). 
Figure 1 Reproduction of Watson and Crick’s 1953 model of DNA (University of Cambridge Museums, 2014).

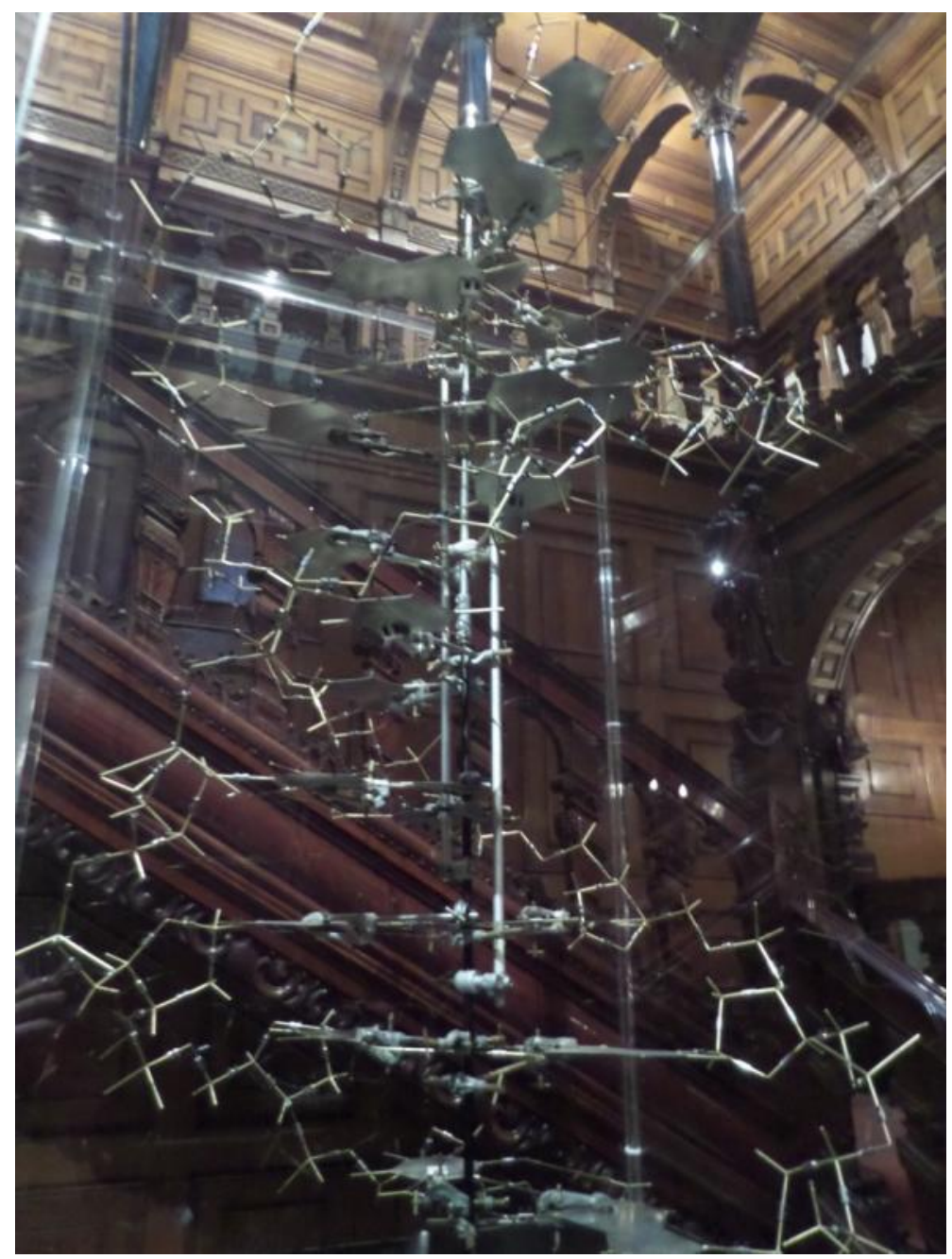

Due to the two-dimension nature of most display techniques, and most significantly historically on paper, the structure of molecules has traditionally been recorded using 2D diagrams consisting of the shorthand letters used for elements and the bonds between them indicated with connecting lines. This did not change significantly between generations of chemists. See for example, Figure $2 \mathrm{a}$ and Figure $2 \mathrm{~b}$, showing the structure of chlorophyll $a$ in two books, published in the 1940s and 1960s (Bowen, 1946; Bowen, 1966). Such diagrams were produced by hand without the aid of a computer. The absolute configuration of chlorophyll $a$ was determined by Ian Fleming in 1967 (Fleming 1967). This allowed the structure to be drawn in a semi threedimensional representation, as in Figure 3b. Nowadays, the structure of such a molecule can be viewed in a much richer set of ways. See, for example, three visualisations available on the Wikipedia page for chlorophyll $a$ in Figure 4 (Wikipedia, 2014a). Even more impressive, such molecules can now be viewed with other larger structures. For example, see chlorophyll $a$ embedded within the major light harvesting complex from spinach in Figure 5. 
Figure 2a Structure of chlorophyll $a$ (Bowen, 1946).

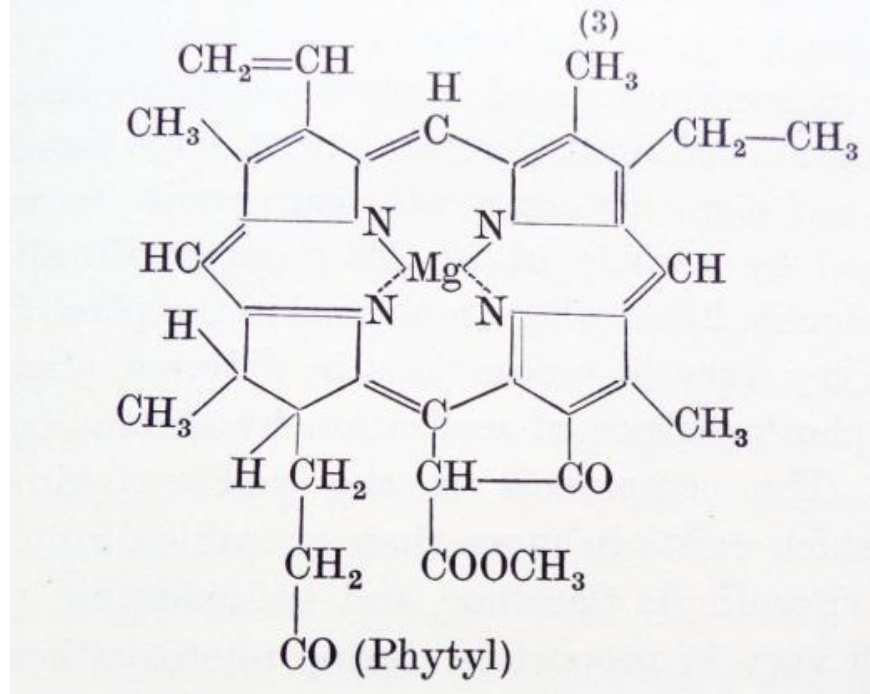

(Phytyl) is

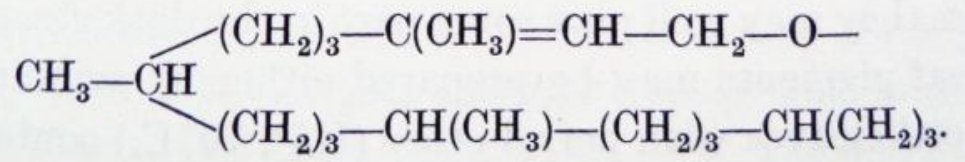

Figure 2b Structure of chlorophyll $a$ (Bowen, 1966).

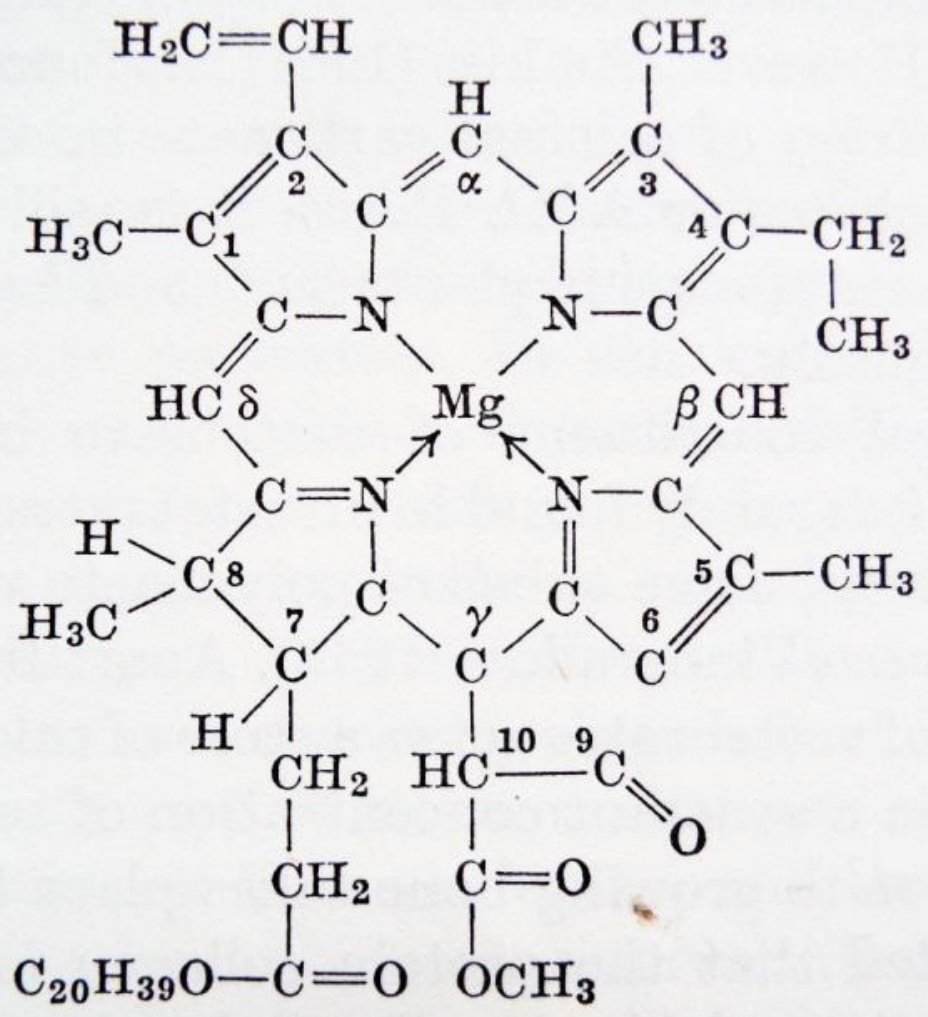


Figure 3 Structure of chlorophyll $a$ (Fleming, 1967). In this representation the bonds shown as bold wedges project out from the page and those depicted as dashed wedges project into the plane of the page; all other bonds lie in the plane of the page. Carbon and hydrogen atoms are not all shown explicitly; carbon atoms occur at the terminus and junction of all bond lines unless another group is shown explicitly and hydrogen are implicit to bring the valence of each carbon to 4 .

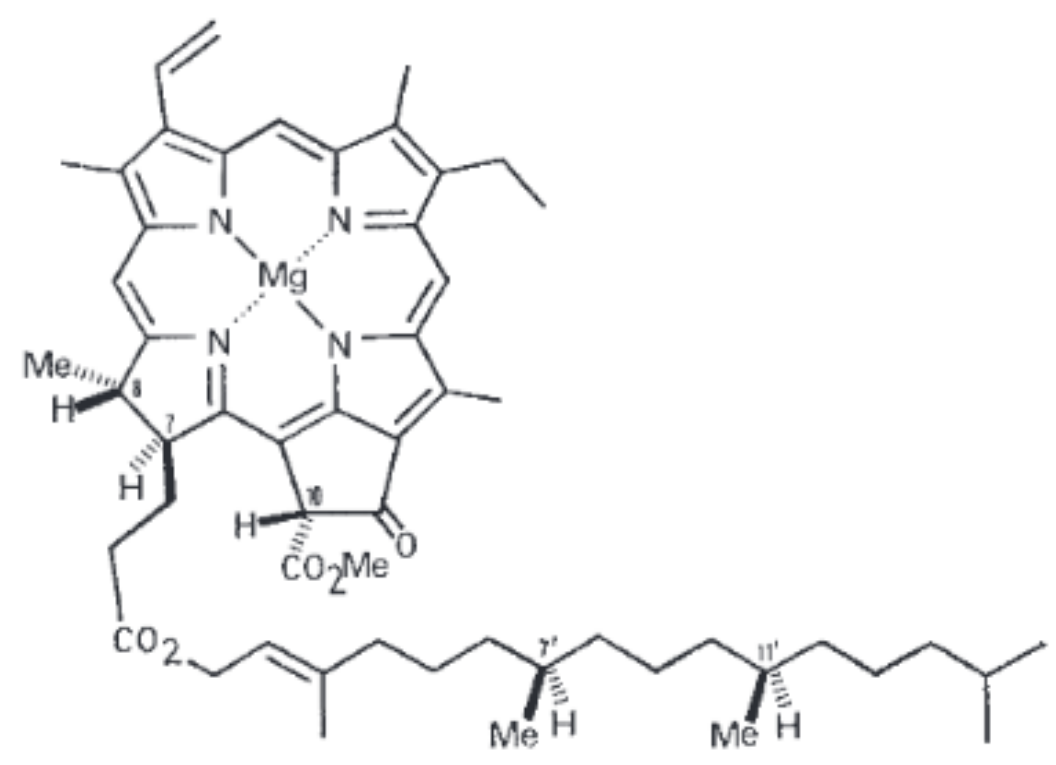

11

Figure 4 molecule.
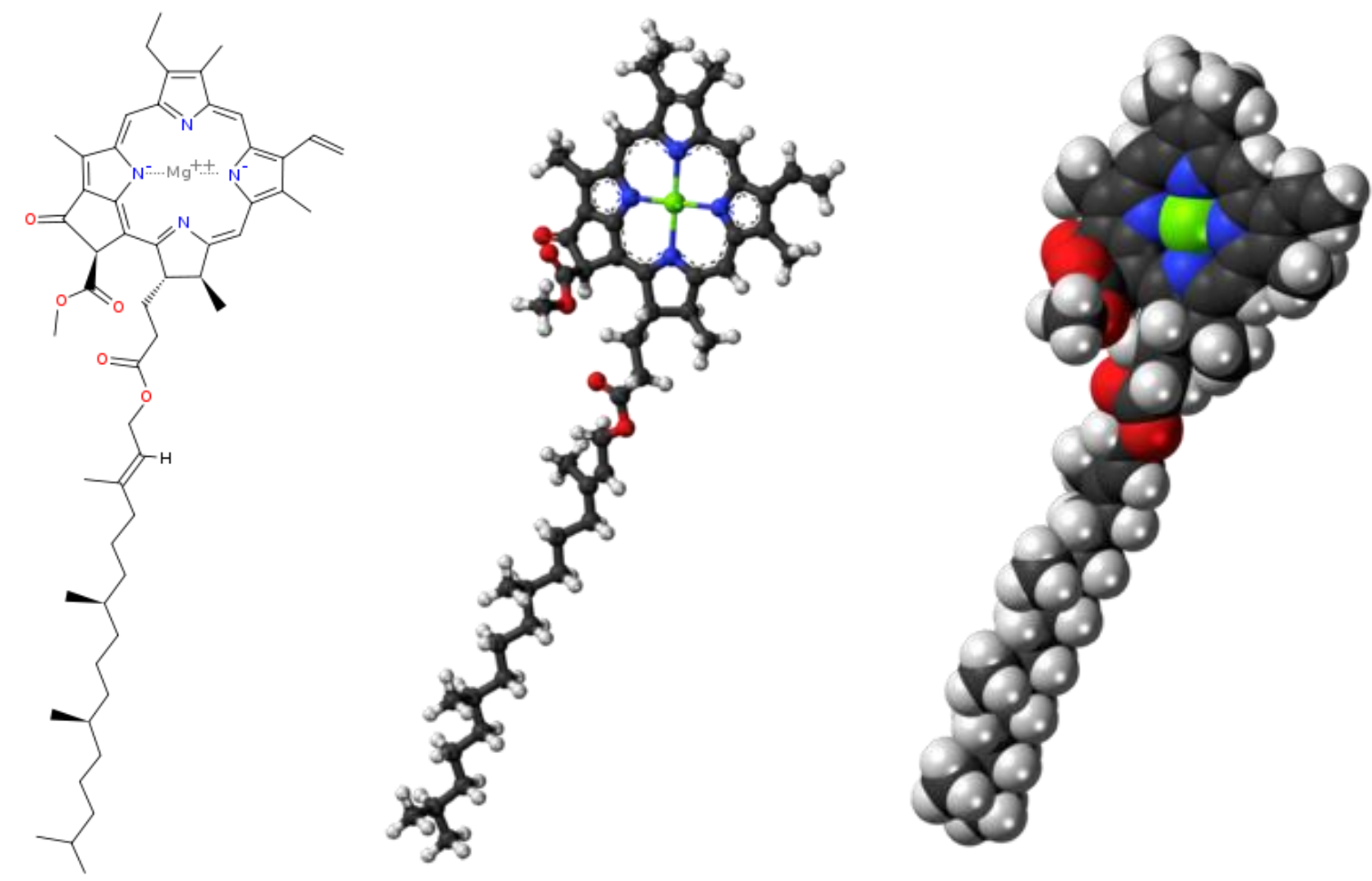
Figure 5 Modern visualisation of chlorophyll $a$ ligands in green in the crystal structure of spinach major light harvesting complex ( $p d b$ code: $1 r w t)$.



Within Figure 5, chlorophyll $a$ ligands are shown in green within the crystal structure of spinach major light harvesting complex (pdb code: 1rwt) (Liu, 2004). The protein backbone is shown in cyan as a ribbon diagram with a transparent protein surface superimposed. The other chlorophyll ligands are shown in white. The image was created using UCSF Chimera (Pettersen, 2004).

As can be seen, such visualisations start to take on an artistic quality, especially as the complexity increases. This has been noted by publishers of chemistry journals, which take advantage of this fact to produce eye-catching covers. This is in contrast to computer science journals, for example, despite the fact that it is easy to use computers to help in the production of interesting artwork nowadays. Compare for example, the selection of journal images, largely covers, for computer science journals in Figure 6a with the equivalent selection of chemistry journal images in Figure 6b (collected using Google Images). It can be seen that the complexity of the graphics for the latter is significantly more complex and arguably more interesting than that for the former. Many of the chemistry journal covers in Figure $6 \mathrm{~b}$ include visualisations of molecules. 
Creative Visualisation in Chemistry

J. P. Bowen, A. M. Bowen, and K. Harrison

Figure 6a Sample computer science journal covers and images (Google Images).
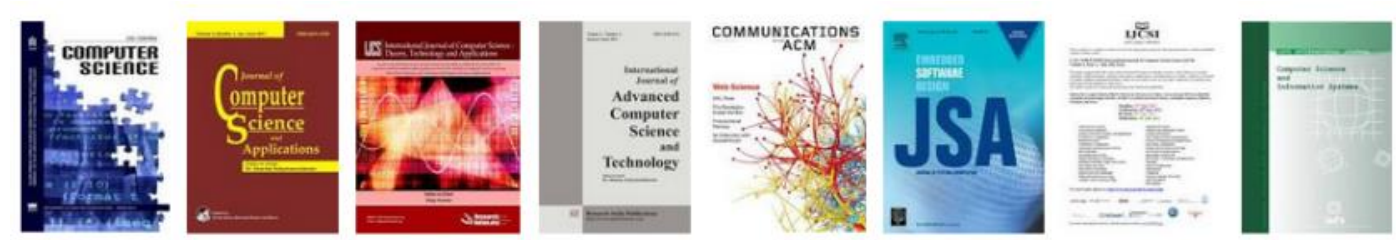

Academy Publish
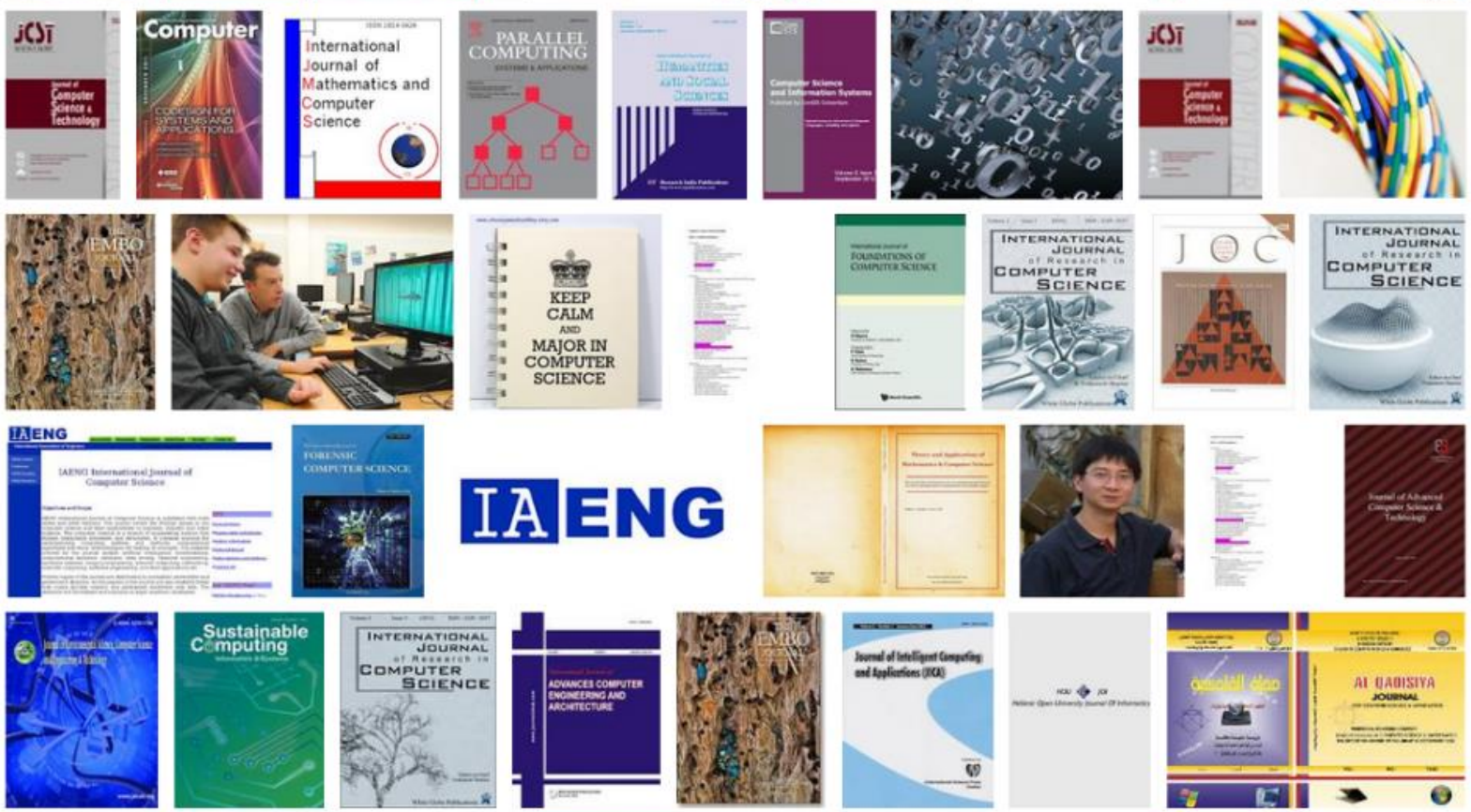

IAENG
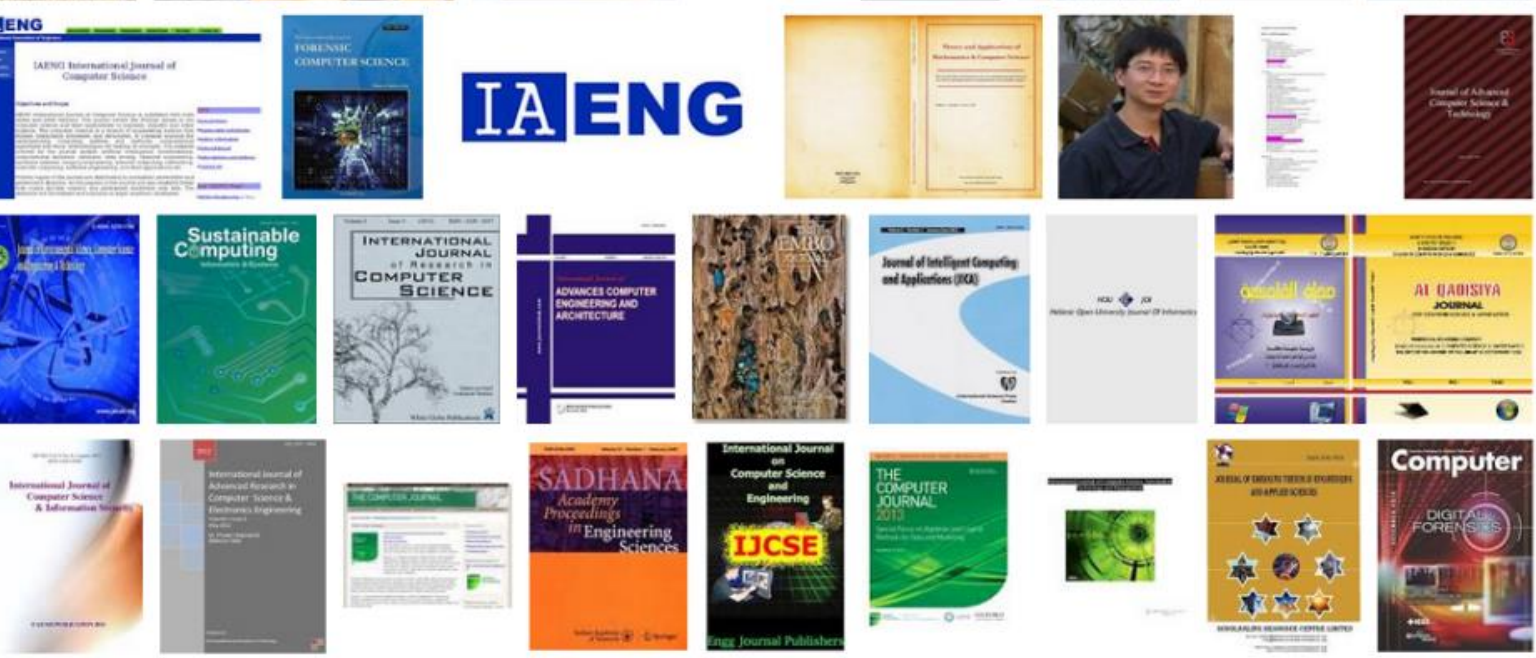
Creative Visualisation in Chemistry

J. P. Bowen, A. M. Bowen, and K. Harrison

Figure 6b

Sample chemistry journal covers and images (Google Images).
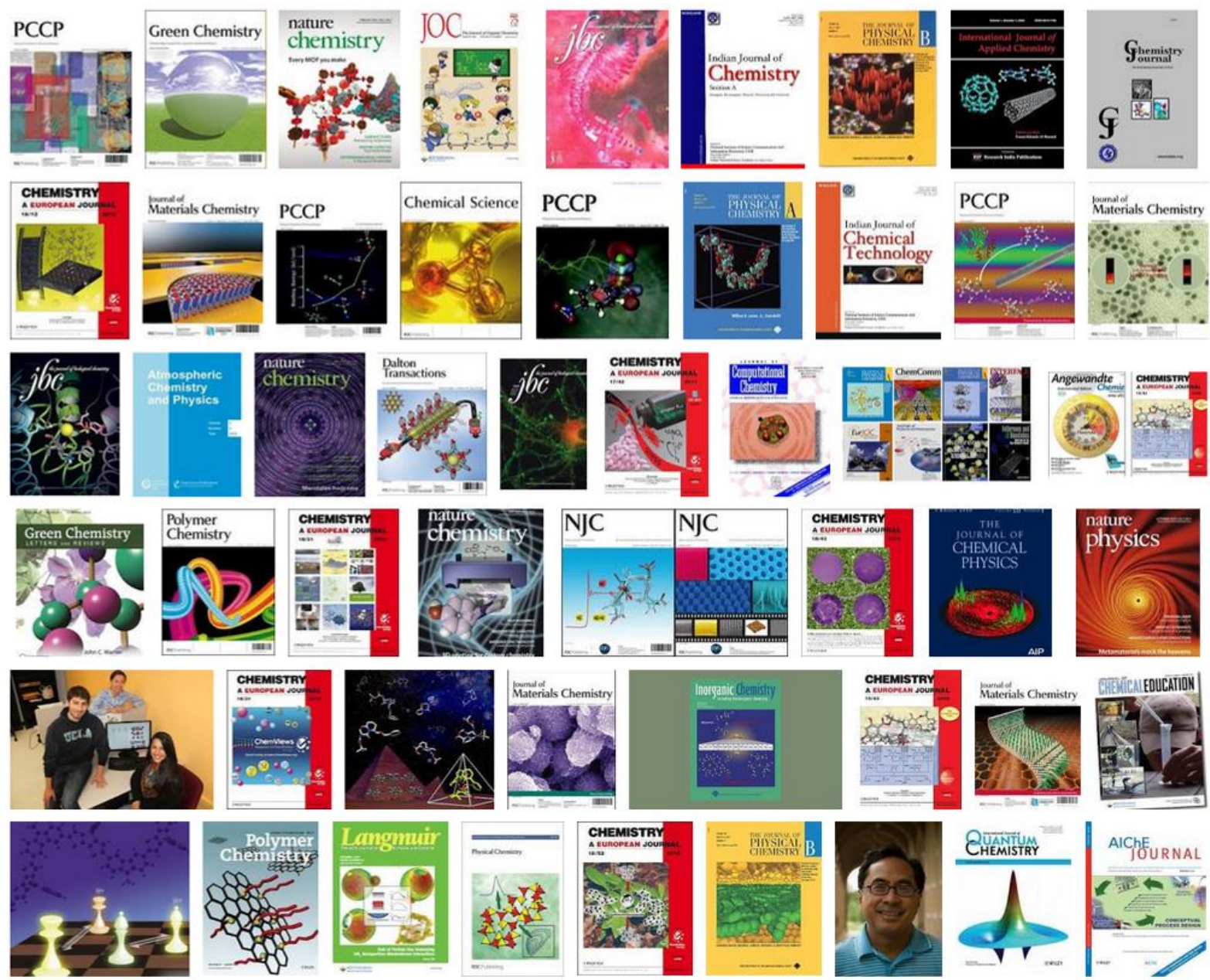
Figure 7 Visualisation of molecules using UCSF Chimera (Wikimedia Commons).

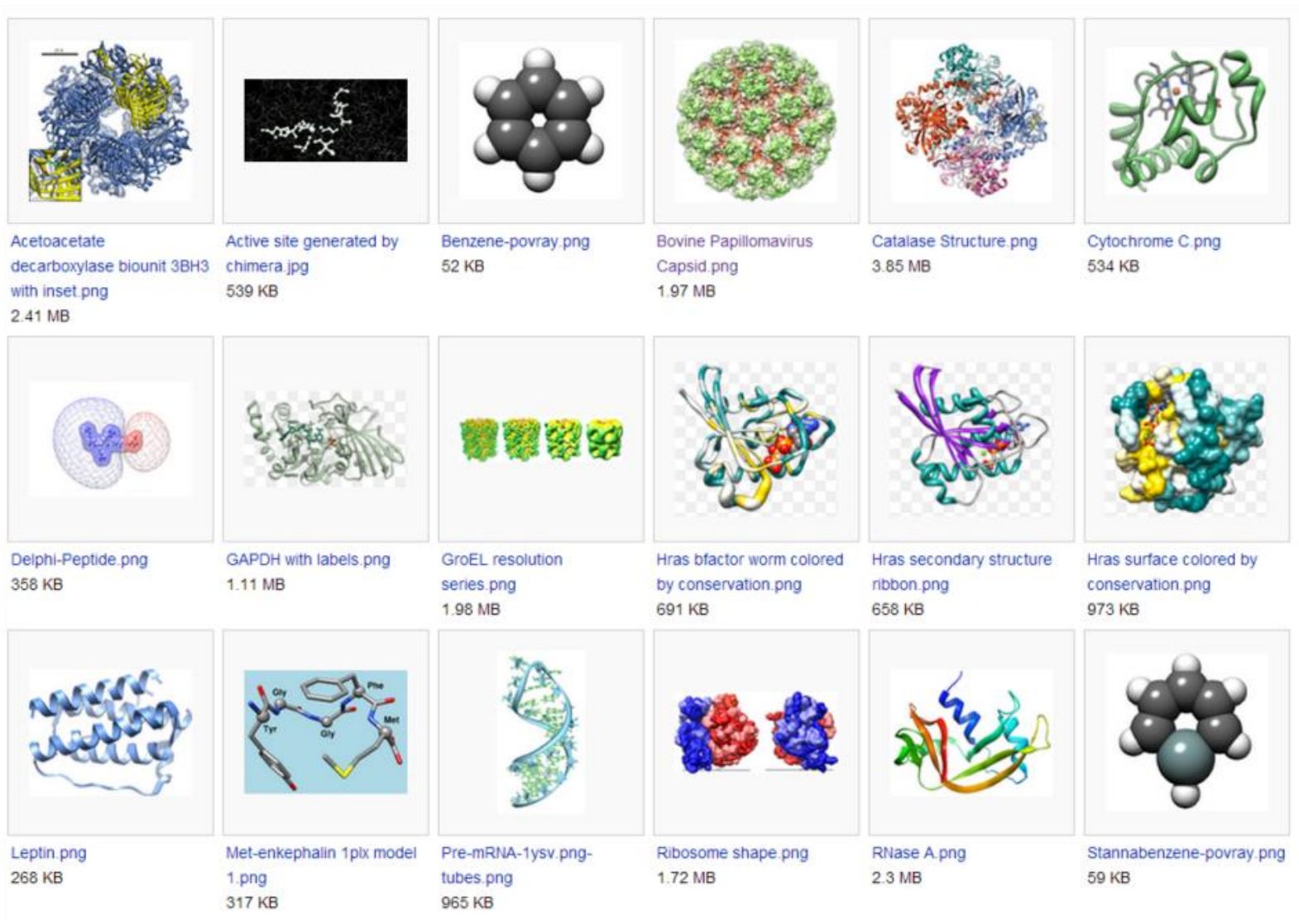

Nowadays, chemists routinely use software as part of their work. For example, virtual chemistry allows chemical reactions to be simulated. In particular with respect to this paper, a selection of software is available for the visualisation of complex 3-dimensional molecular structures. Many of these are very beautiful in their own right. As well as being included as illustrations in academic papers, such visualisations are often used on the covers of chemistry journals as artistically decorative and attractive motifs (as illustrated in Figure 6b). Some such images are freely available for use by anyone. See for example the selection of images produced with the aid of the widely used UCSF Chimera software on the Wikimedia Commons website in Figure 7. Chemical images have also been used as the basis of actual artworks in exhibitions as well as for artistic illustrative purposes.

The rest of this paper explores the development of the relationship of chemistry, art, and IT in a historical context, especially with respect to the production of chemistry journal covers using computer-base visualisation techniques. It also gives an overview of some of the increasingly sophisticated software used to generate these projections.

\section{History}

From the very beginnings of the understanding of chemical structure, chemists have had to use visualisations to represent their view of their science. Chemistry is the creation and study of the properties of molecular and chemical structures built from the bonding of atoms, these atoms are composed of infinitively small atomic nuclei and clouds of electrons in probability fields. Bonds are formed from the overlap of these electronic clouds of quantum distribution. None of this can be seen explicitly and only inferred from experimental results such as the perturbation and diffraction of X-ray or neutron beams. As a result chemists have devised schematics to illustrate the chemical properties and reactions of the molecules they study. These schematics of course are used in the research publications and textbooks to explain and discus chemistry. Originally, chemical schematics could be produced using typewriters and simple offset printer plates (Figure 8). 
Figure 8 Typeset chemical structures (Grundon, 1962).

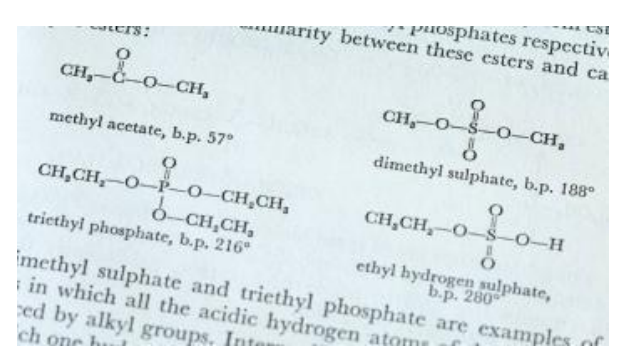

Quite rapidly, these simple representations of chemical structure became unsatisfactory as the knowledge of chemical structure evolved. Many shorthand structures were developed to allow chemists to describe three dimensional structures on a two dimensional plane; some examples are shown in Figure 9.

Figure 9 Two dimensional projections drawn on ChemBioDraw Ultra V 12.0 the types of projections are described in detail in IUPAC (1997).

Staggered conformations

Eclipsed conformations

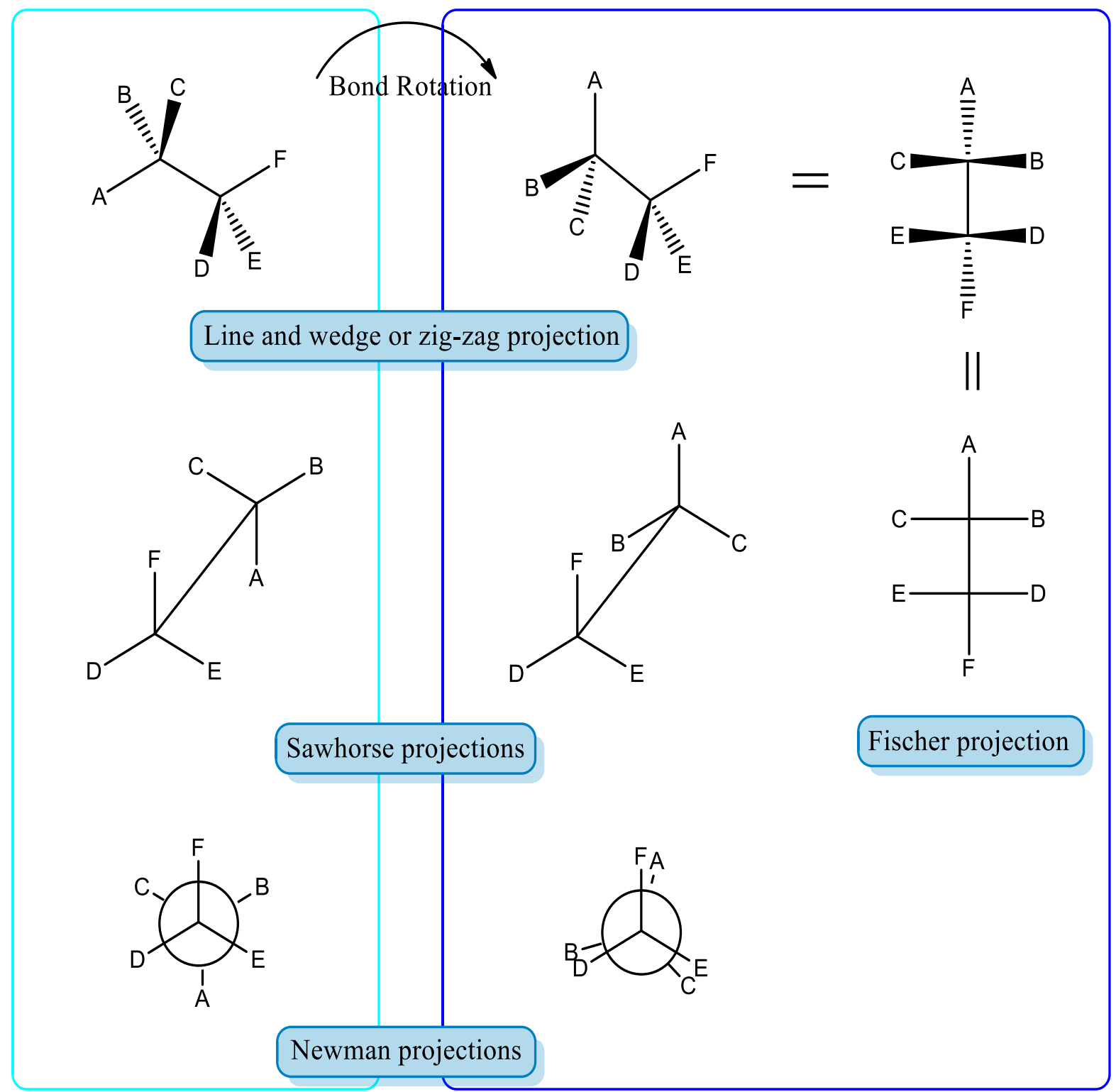


For more complex structures, illustrators became employed either at publishers or even embedded into science departments to produce the schematic illustrations for the science community (Figure 10 and Figure 11).

Figure $10 \quad$ Early illustration of structures (Wells, 1956).

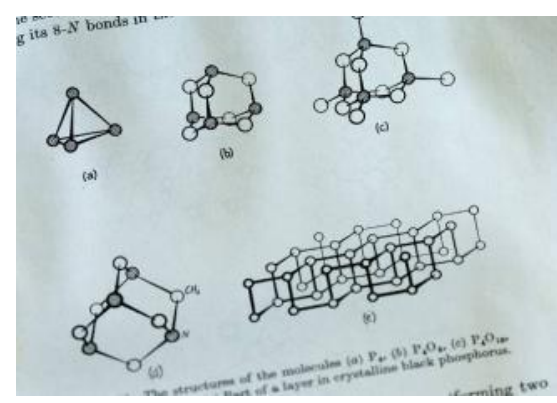

Figure 11 Representation of chemical to protein interactions (Goodwin, 1964).

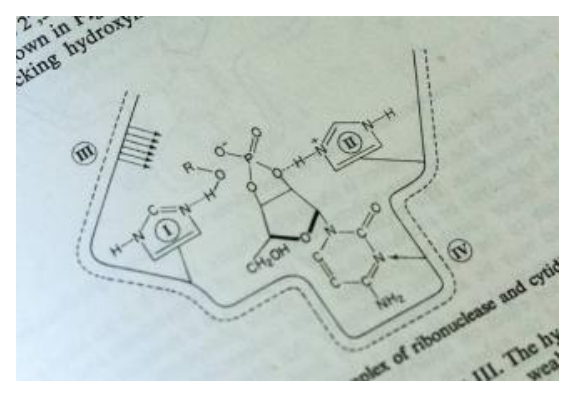

One of most famous illustrators in this field was Irving Geis, who worked with publishers to illustrate chemistry and biochemistry structures. In 1961, he was commissioned by Scientific American to illustrate John Kendrew's article of the first protein structure, Myoglobin, followed by illustrations for the article by David Phillips (1966) on the first enzyme structure, Lysozyme (Figure 12).

Figure 12 Painting of Lysozyme (Philips, 1966). Many modern software packages use similar visualisation techniques to this illustration, showing just how advanced this illustration was for its time.

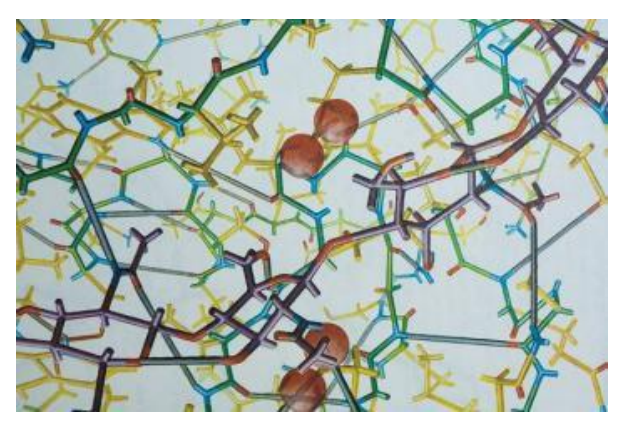

In 1976 Irving Geis co-authored a beautiful textbook with Richard Dickerson entitled Chemistry, Matter, and the Universe (Dickerson \& Geis, 1976). See Figures 13 and 14. 
Figure 13 Organic alkane chains (Dickerson \& Geis, 1976).

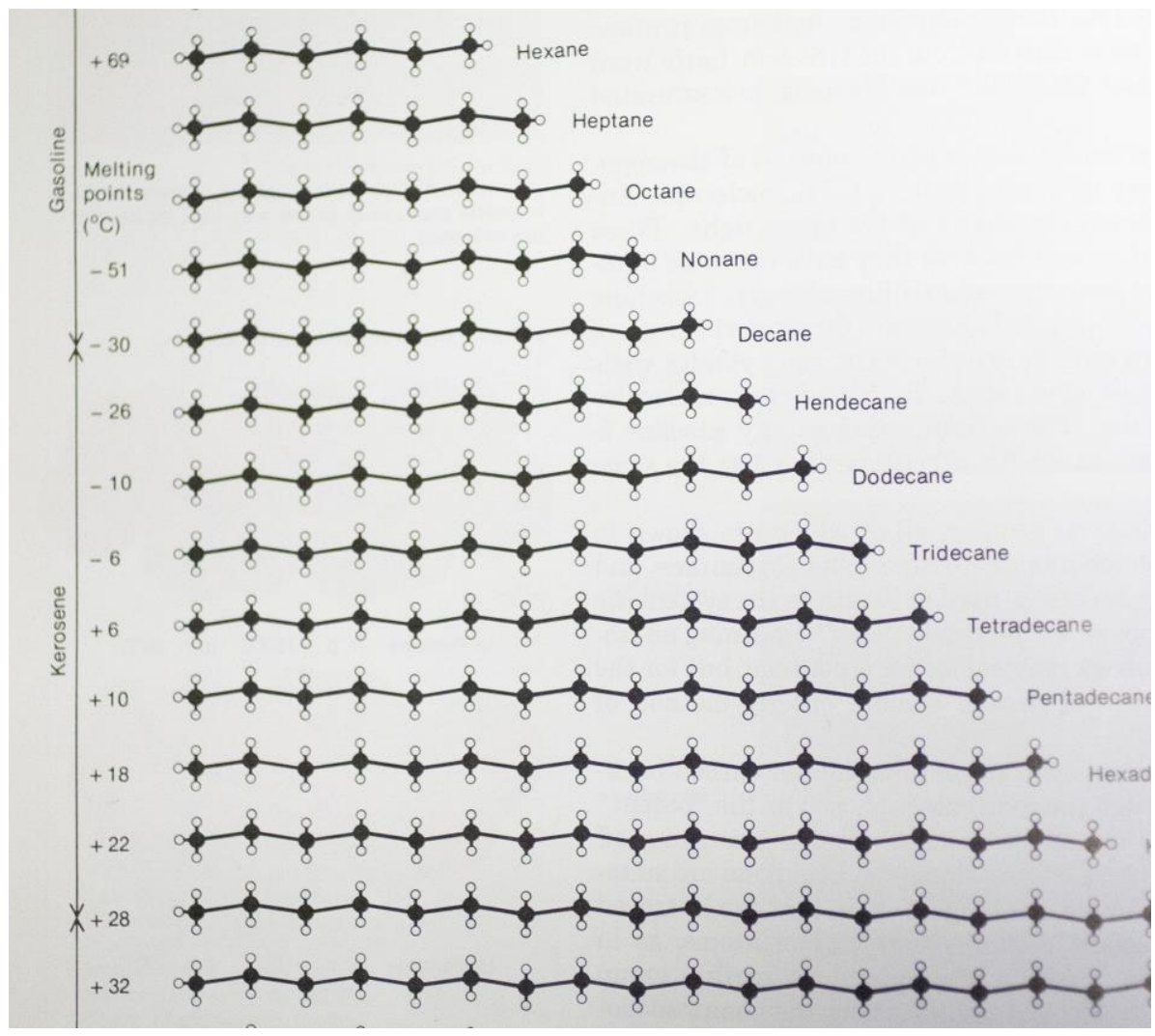

Figure 14 The chemistry of soap bubbles (Dickerson \& Geis, 1976).

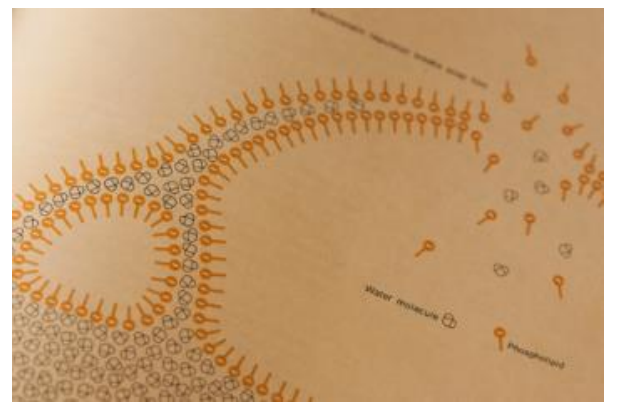

However the rapid rate of chemistry publications and growth of scientific knowledge resulted in the chemists needing to produce camera-ready illustrations themselves for their research papers and books.

So from the late 1970 s to mid 1980 s, many science authors became expert using Rotring pens ${ }^{\text {TM }}$, plastic templates stencils and Letraset ${ }^{\mathrm{TM}}$. Thus many hours would be spent by chemists, not trained as illustrators, trying to produce camera-ready illustrations using these tools (Figure 15), with a constant worry of smudging the ink or tearing a piece of Letraset ${ }^{\mathrm{TM}}$ transfer that could force them to start over again. 
Figure 15 Chemistry illustration tools.

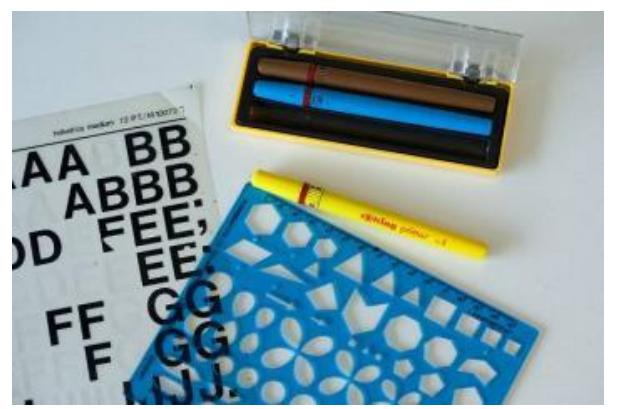

Apple introduced the Apple Macintosh personal computer in 1984 and this was revolutionary for chemistry visualisation. The original Apple Mac came with a grey-scale screen and a functioning mouse to allow drawing. It was shipped with MacDraw and MacPaint. Most importantly however, the first Apple Mac came with the ability to print Adobe PostScript to the very first Apple laser-printer. This was a game-changing innovation; for the first time, a computer could be used to produce illustrations ready for print and publication

In 1986, Cambridgesoft was founded and released ChemDraw for the Mac. This rapidly prompted chemists around the world to buy Apple Mac computers and to this day desktop computing in some chemistry departments is still dominated by Apple Mac computers. In 1994, Cambridgesoft released ChemDraw 3.1 for Windows, and this allowed the then new Microsoft Windows computers using MS Windows 3.1, to have a chemistry drawing solution for their computer platform. Cambridgesoft's ChemDraw is still very much the publishing standard and many journals will only accept chemical illustrations produced in this propriety file format. Complex chemical schematics and illustrations are rapidly produced in ChemDraw and it has inbuilt chemical intelligence knowing structural rules, error highlighting and auto-drawing/correction functions from formulae, structural names or even poorly drawn figures (see Figure 16). 
Figure 16a Chemical drawings of sucrose, C60 and Taxol.

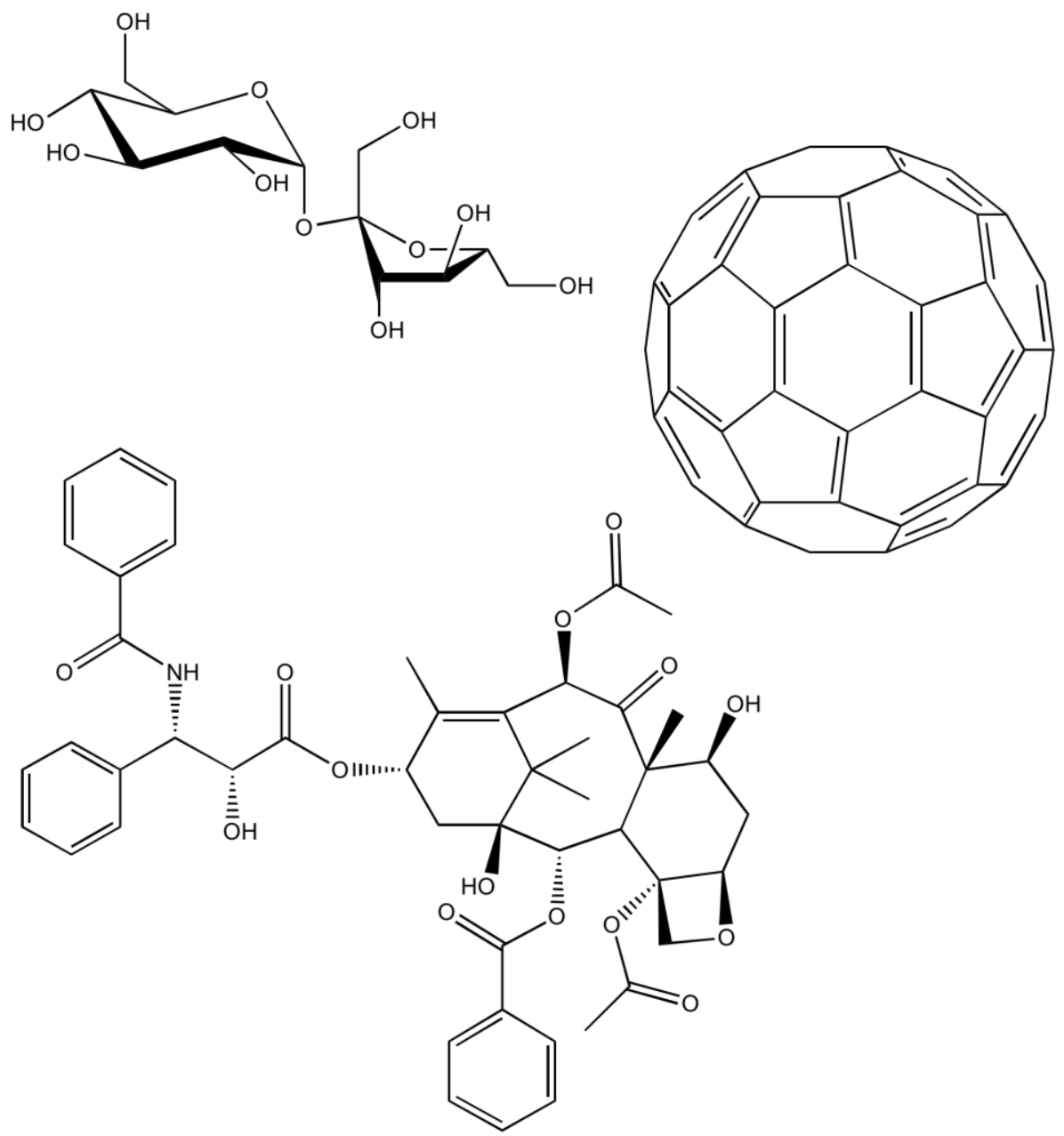

Figure 16b An example of the functionality in ChemDraw to correct a poorly drawn chemical structure. Left: figure before correction. Right: figure after correction.
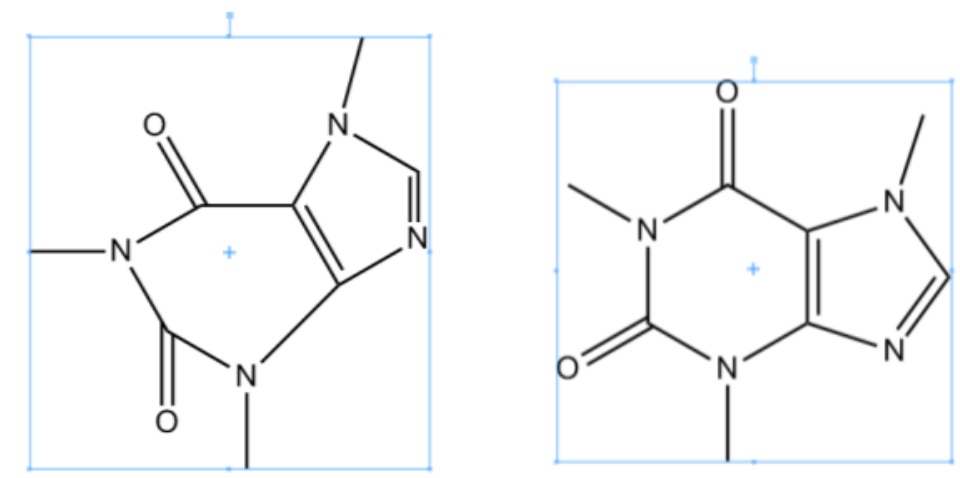

Chemistry though is not just study of two-dimensional line drawings but very much a three-dimensional subject. Although 2D drawings can infer some 3D structure, as shown in Figure 3, traditionally the 3D aspects were covered by tactile 3D models. Originally these were made in wood, then metals and most commercially available modelling kits now use plastic atoms and bonds (Figure 17). 
Figure 17 Metal and plastic models of chemical structures.

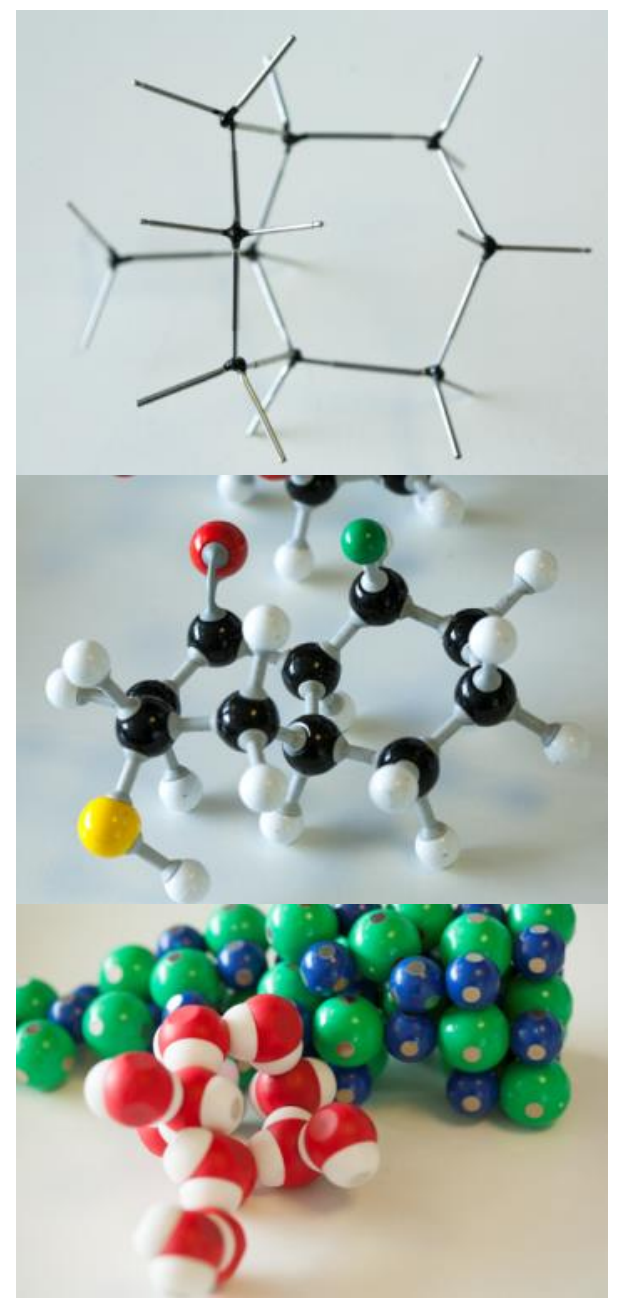

\section{Visualisation}

Chemistry software was quickly produced as soon as desktops computers became popular to provide chemists the ability to visualise their structures in 3D on their computer screens. In 1986, Cambridgesoft released Chem3D, and rapidly there were many other products on the market such as RasMol, Macromodel, Chemical Design, Hypercube, CAChe Scientific all coming in that year. These computational software solutions have grown into a multi-million pounds industry with many vendors and solutions available to the chemists.

This software has enabled chemists to visualise their structures in the many creative ways. The following Figure 18 highlights a collection simple to complex inorganic structures; these inorganic structure illustrations examples have been made from a range of elements across the periodic table.

Figure 18 3D renders of a collection of inorganic chemical structures.
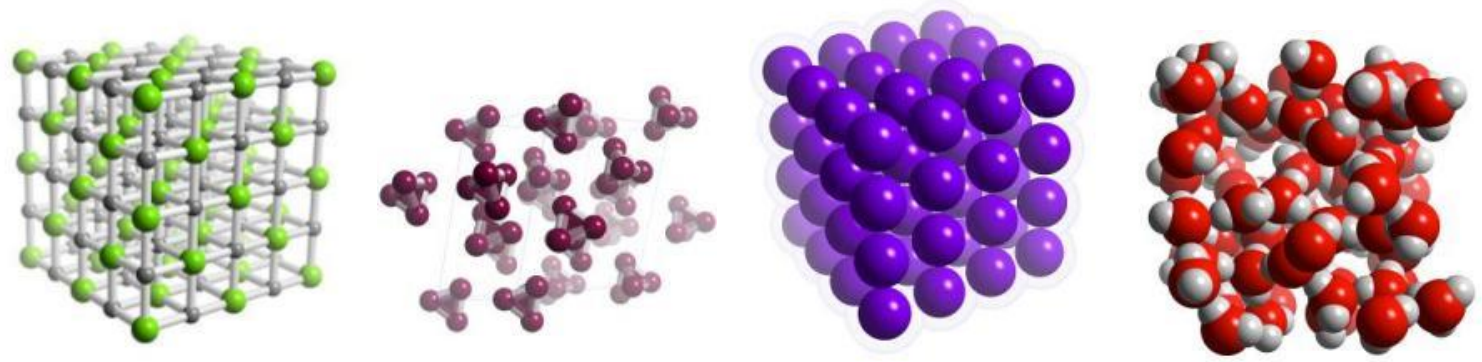

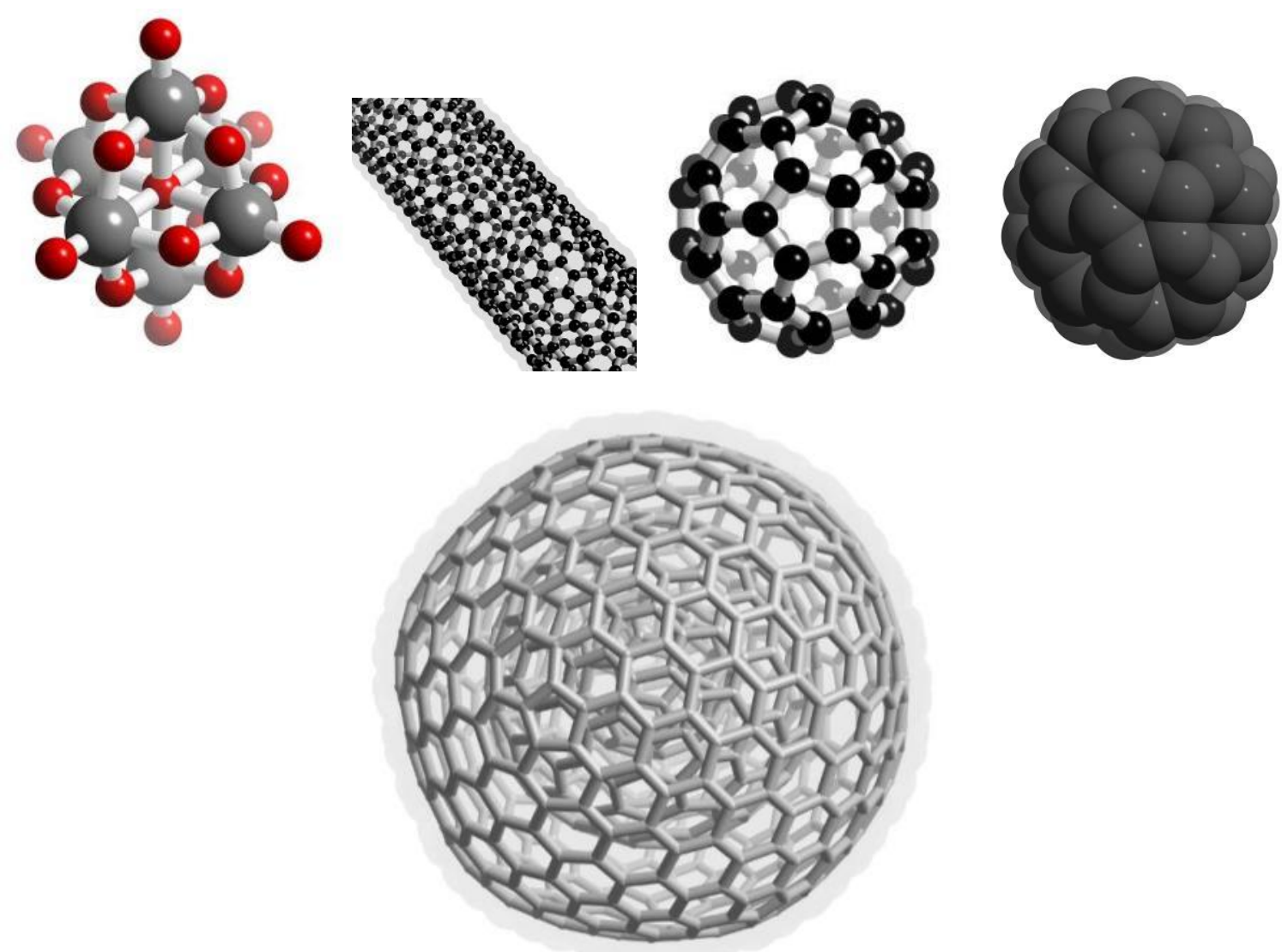

Organic chemical structures made from predominately carbon and hydrogen can be equally beautiful as shown in the following Figure 19.

Figure 19 3D renders of various organic structures.
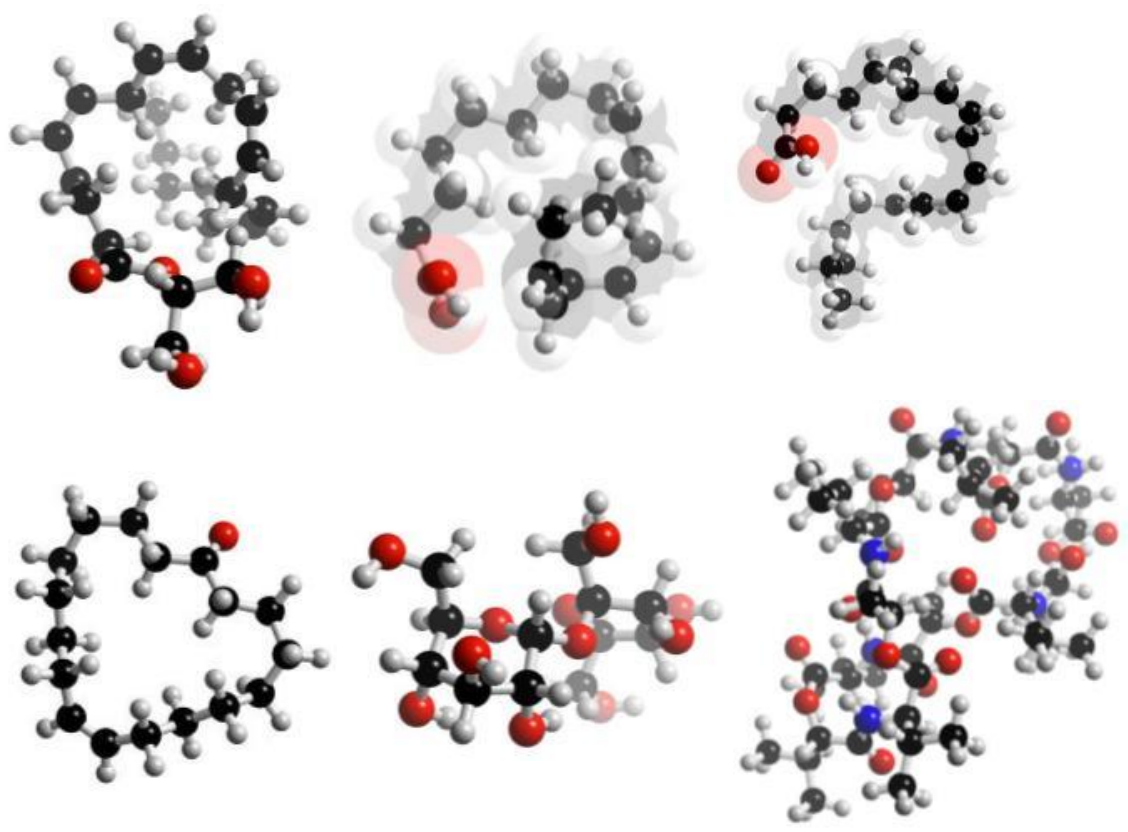

The shape and size of the actual bonds and atoms can be controlled to provide structural information. Figure 20 presents sucrose as a wire frame, a stick model, a ball and stick model, a scaled ball and stick model and finally a space-filling model, also known as a calotte model, is a type of three-dimensional molecular model where the 
atoms are represented by spheres whose radii are proportional to the radii of the atoms and whose centre-tocentre distances are proportional to the distances between the atomic nuclei, all in the same scale.

Figure $20 \quad$ Atom and bond size control in illustrations.
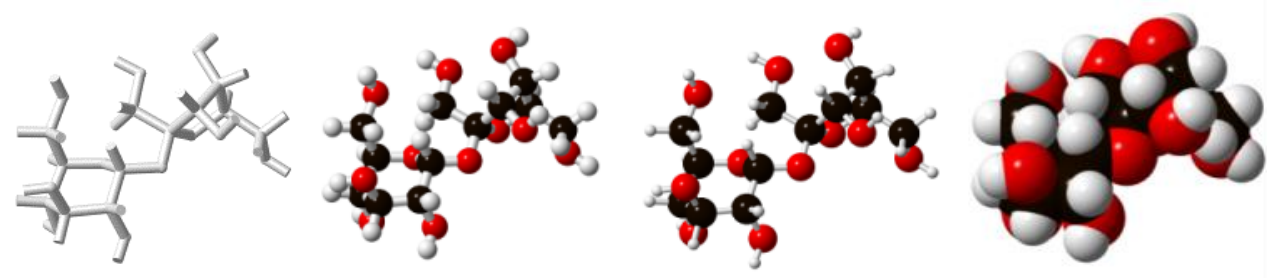

An interesting concept is the use of colour. Of course, colour provides a useful feature in the illustrations to indicate which elements are present in the image without having to label the image. There is the question of what colour should be used. Most chemists recognise a red atom to mean oxygen - but why red? What about hydrogen white and nitrogen blue? We know perhaps carbon is black. Did this colour choice come from the fact that graphite is black, but diamonds, which are solely made from carbon, are not black? How about phosphorus, it is red from the form red phosphorus or white from white phosphorus? Sulphur is easy since that element is only a pure yellow.

In the end, there turns out to be no standard convention and it is up to the illustrator to at least try to be consistent and at some point provide a label if they were to say change oxygen to purple and carbon pink. Certainly chemists are trained to look at the connectivity and recognise the elements based on what they are connected to and their patterns, but changing to "non-standard" colours is generally not helpful. The periodic table can be displayed and collection of spheres and this figure shows a standard choice of colours from Chem3D (Figure 21).

Figure $21 \quad$ Left: Periodic table depicted by spheres showing the relative sizes and usual colours of elements used in Chem3D. Right: Traditional layout of the periodic table (Wikipedia).
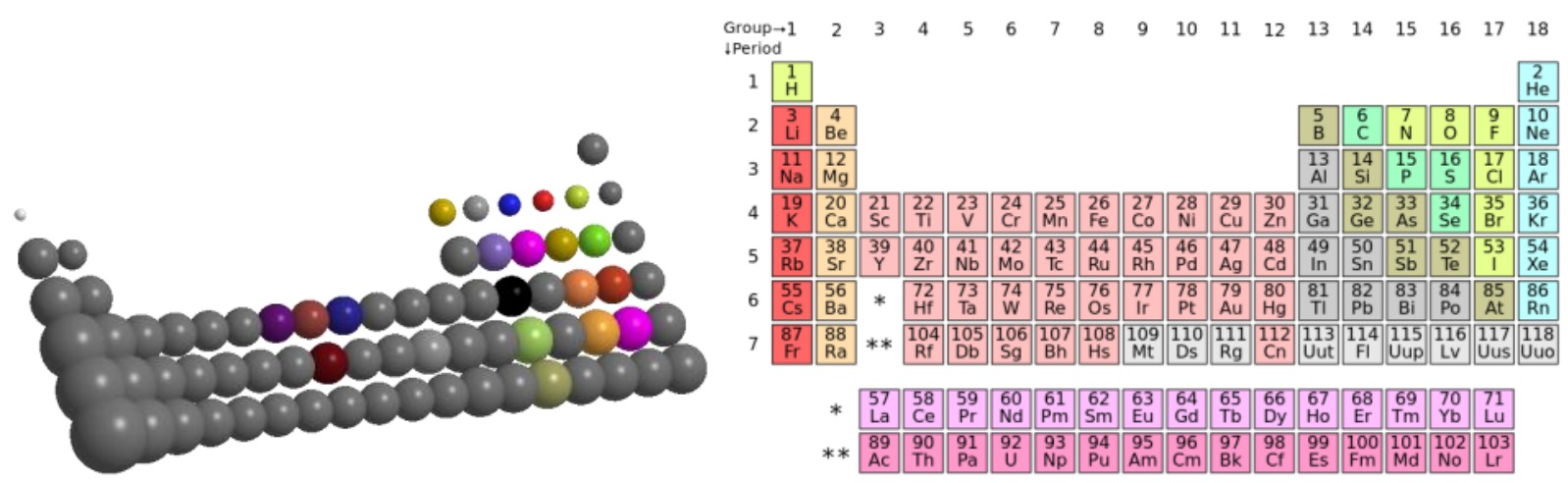

Another aspect at looking at 3D molecular structures is the concept of "secondary structure", inorganic chemists use polyhedral to provide information about repeating patterns. This can be seen in the following example, which has a wire frame, ball and stick view, atom packing view and then polyhedral view of the same structure. The polyhedral view simplifies and exemplifies the repeating network of connections in this inorganic compound (Figure 22). 
Figure 22 Inorganic secondary structure, seen in four different views: from left to right wire, ball and stick, sphere and polyhedral.
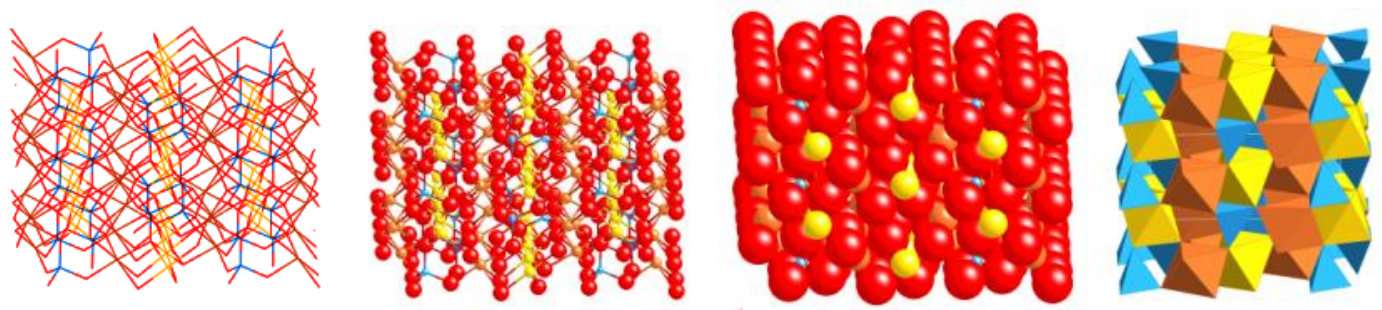

The following other inorganic polyhedral views shows the natural beauty these structure can exhibit (Figure 23).

Figure $23 \quad$ Inorganic polyhedral illustrations.
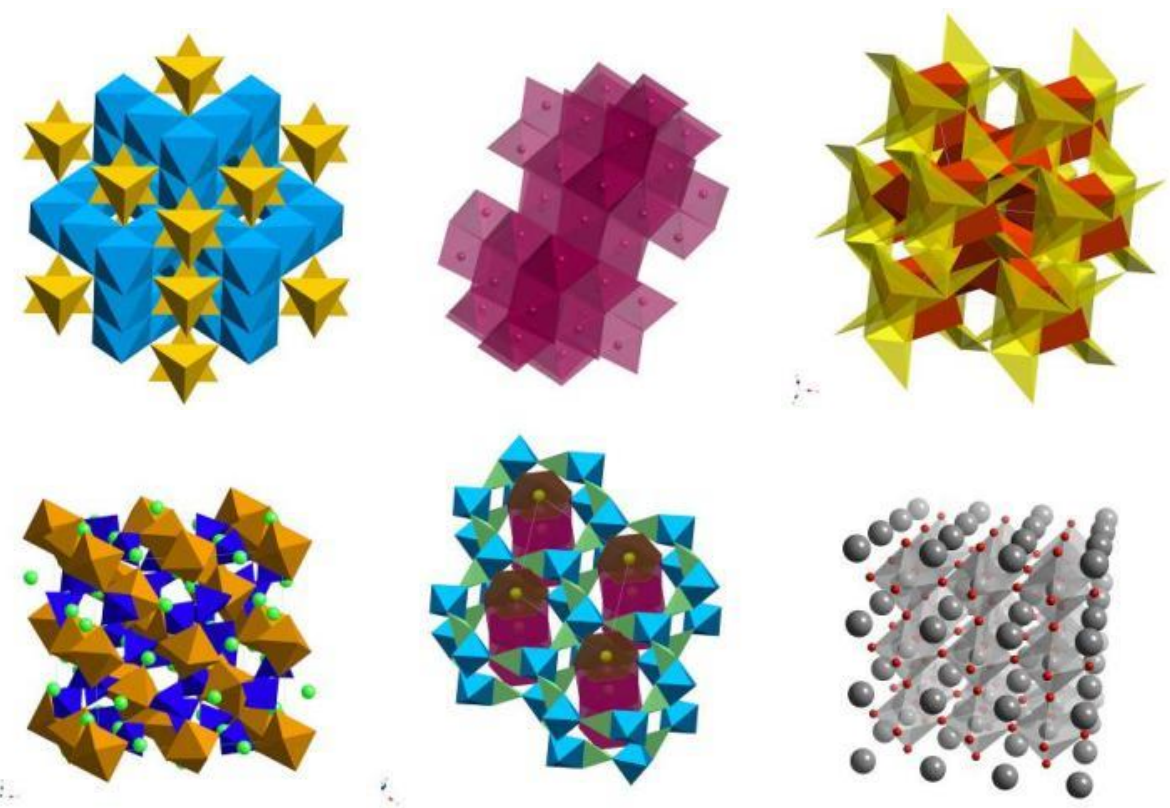

Of course, not to be outdone, biological structures have a "secondary structure" repeating structural motif to describe how the amino acids or nucleic acid base pairs join together to form a large 3D structure. The most famous of these is the double helix of DNA (Figure 24), where the two strands of DNA bind together with complementary base pairing to form a ladder of pairs between the two spiral stands of DNA. Looking at the resulting separation of the helices in the secondary structure, one can see the major and minor groves - wider and narrower spacing - which leads to structural properties found in DNA chains and the enzymes that bind to them. 

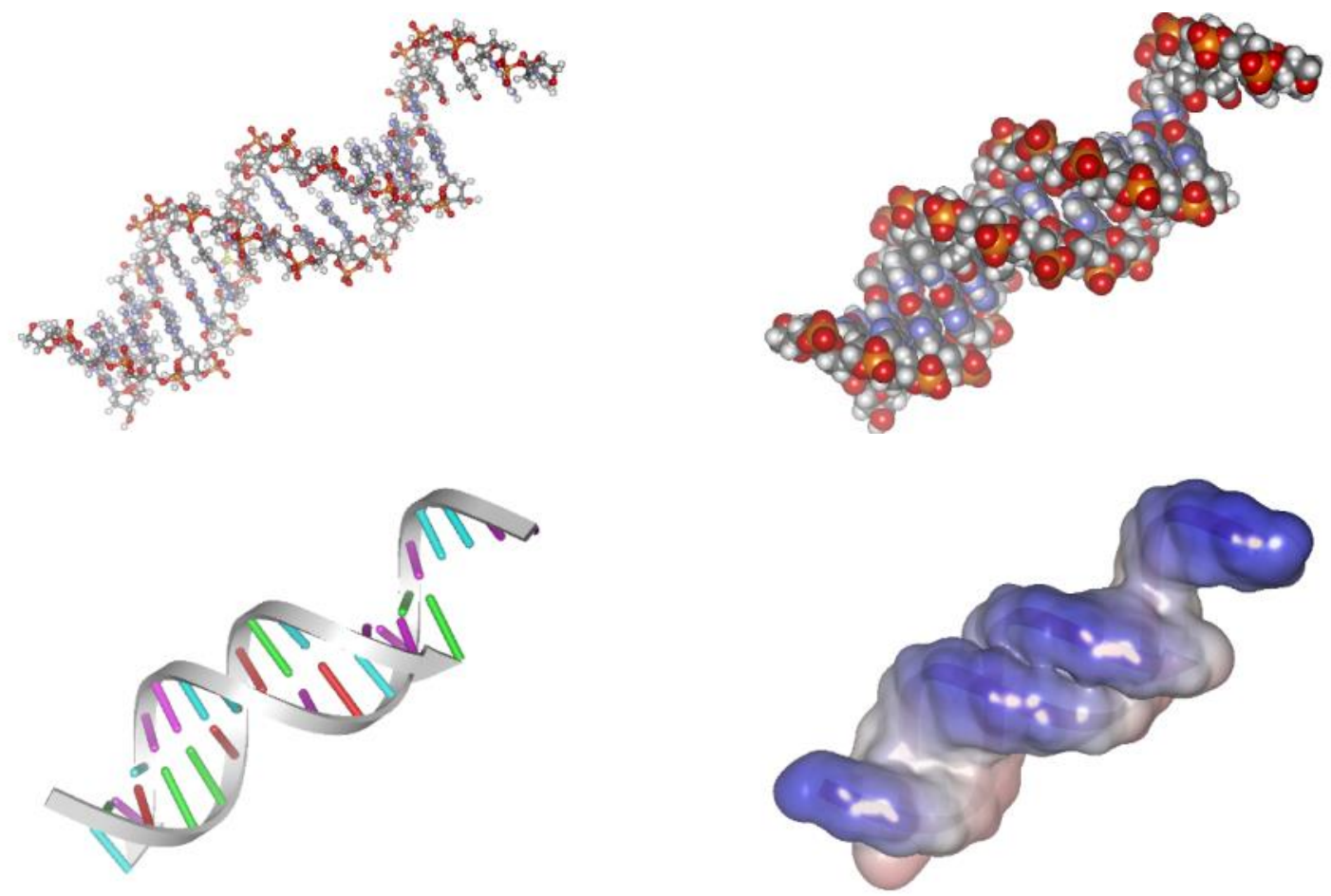

Proteins and enzymes themselves have secondary structures based on the amino acid sequences where certain sequences of amino acids give rise to sheets and others give rise to helices in the secondary structure views. It is the combination of helices and sheets which gives rise to the folded pattern which in turn provides a unique shape the protein or enzyme adopts and thus the biological chemistry that these structures perform (Figure 25).

All these colourful illustrations and 3D images that the portfolio of chemistry software packages has produced have graced in the insides of the chemistry textbooks and research publications for the last twenty years. Some modern protein visualisation software contains algorithms to select the regions of the protein that can be classified into helices and sheets automatically; other visualisation packages extract this information, when it is present, from the protein structure file.

Figure 25 Protein and enzyme secondary structures. Alpha helices are shown in red, beta sheets in yellow, and loop and linking regions where the structure is less defined are shown in green.
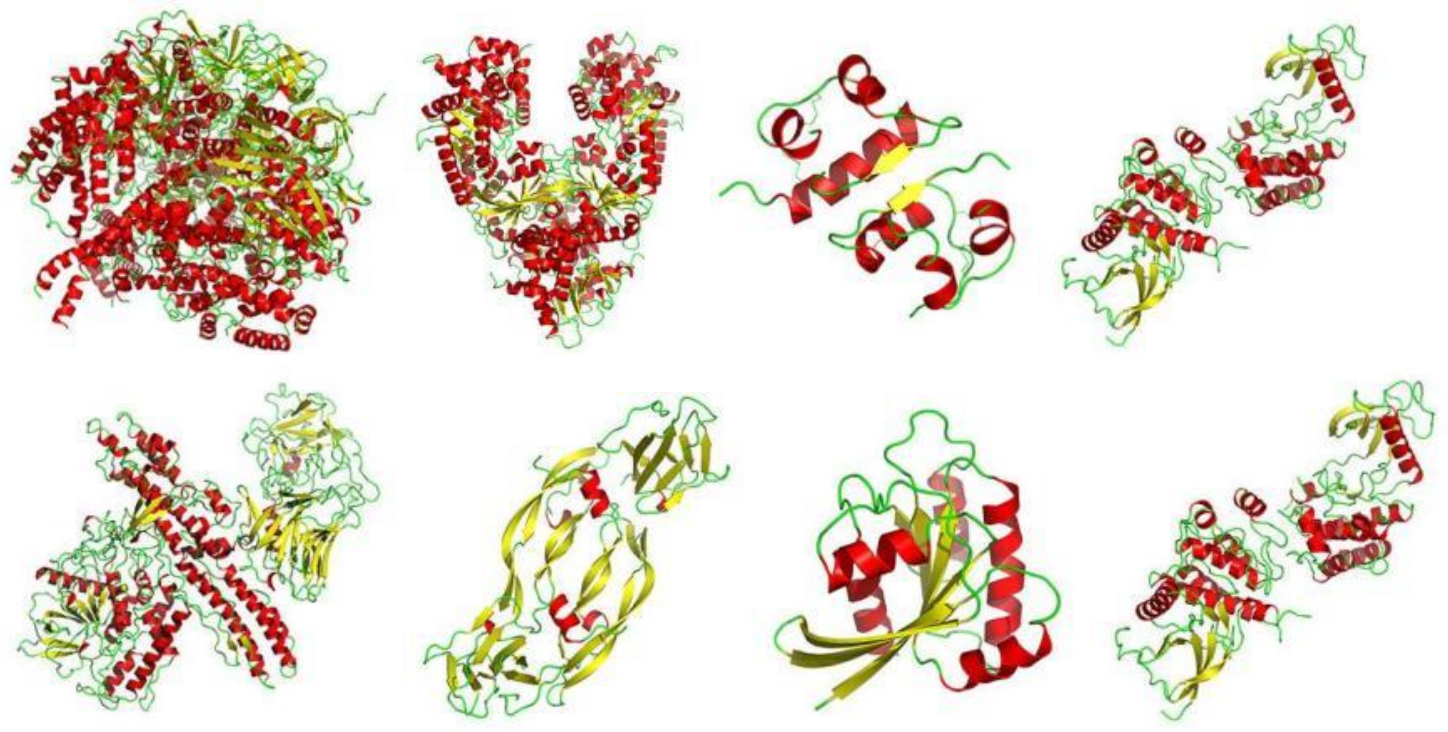


\section{Journal Covers}

More recently, journals and textbooks have been taking these colourful illustrations and expanding upon them for use as journal cover artwork (Figure 26).

Figure 26 Example journal covers with chemistry art.

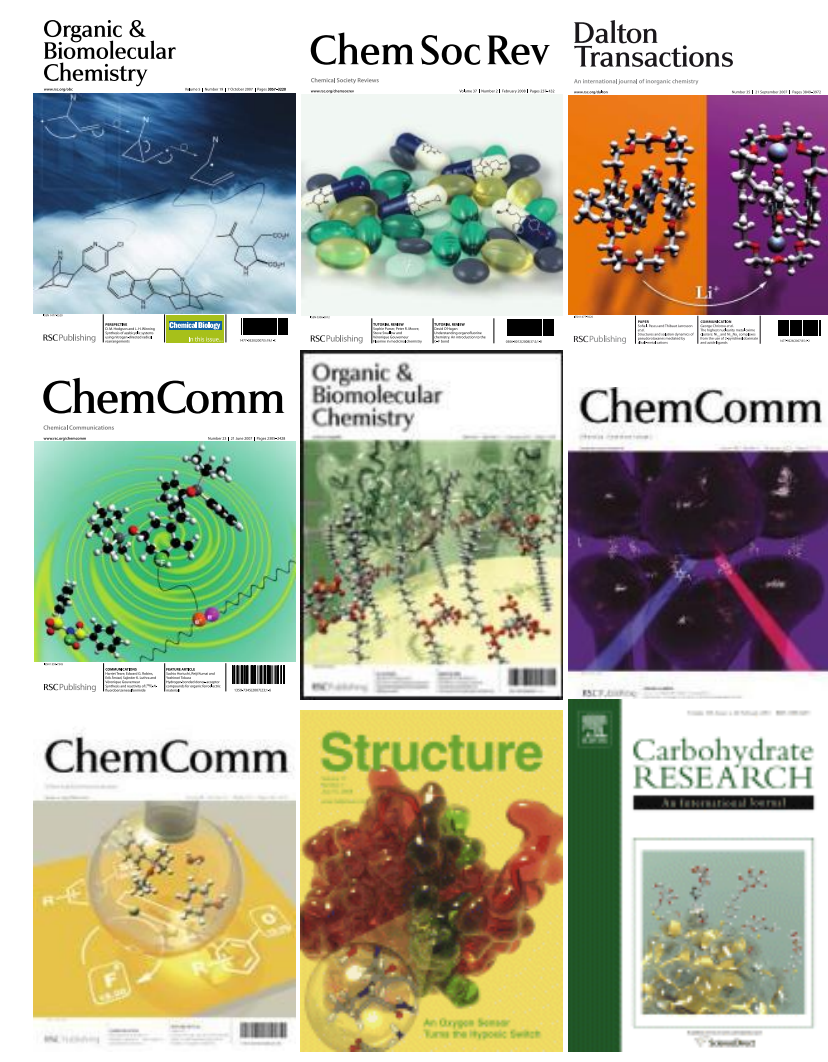

There are number of interesting comments to say regarding this process. Commercial or even learned society journal publishers will often give an invitation saying that an article was very well received by the reviewers and the editorial office, so they would like to invite the authors to submit some striking artwork for consideration for use on the cover of the issue in which the article will appear. Of course, the author(s) may flattered by the request and wish to supply an image to promote their science.

A subsequent comment from the publisher then typically mentions that if the artwork is chosen then the authors will be asked to make a contribution towards the production costs. This cost can run into many hundreds of pounds for the scientist to submit a cover figure. When the number of journal titles a publisher has on offer is considered, together with the frequency that these titles are produced, the publisher has a significant additional income stream of potentially several hundreds of thousands of dollars per annum. It is an open question as to whether scientific or financial considerations are more important to publishers. 
Figure 27 3D render lightning effects on a simple organic molecule.

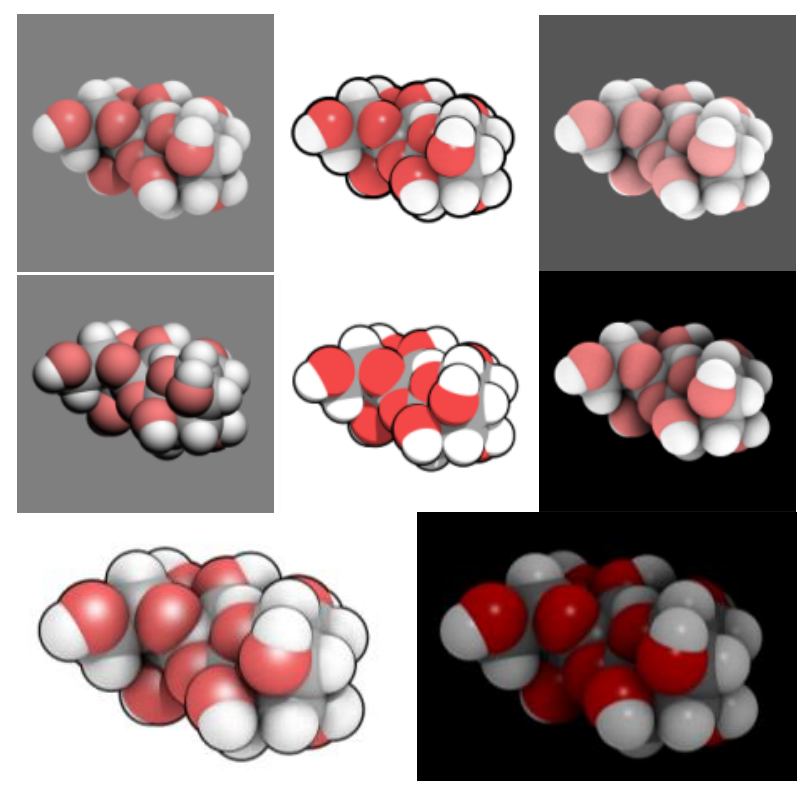

Figure 28 3D render lightning effects on a protein.
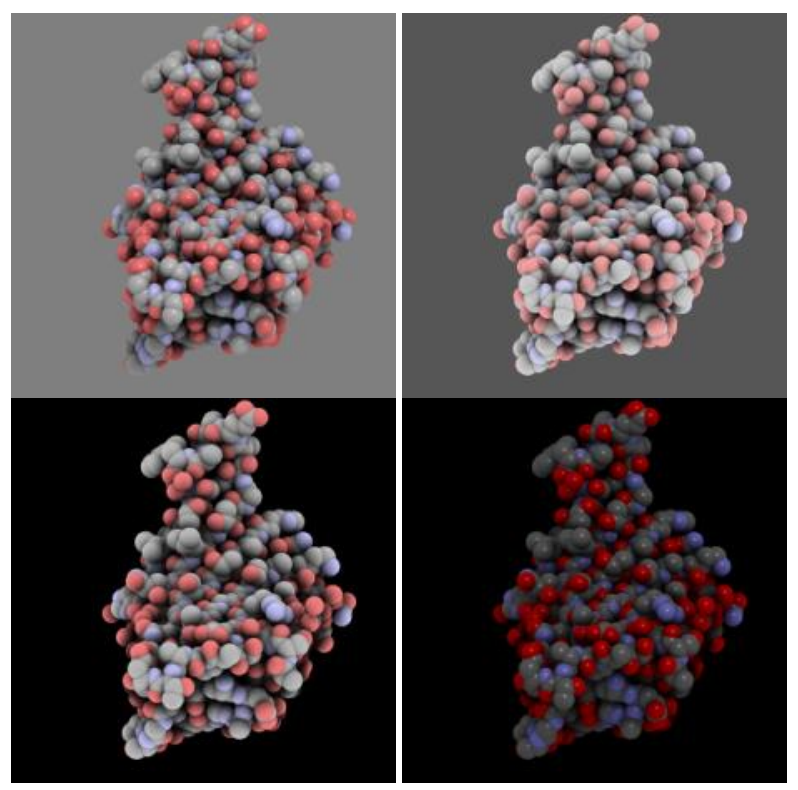

The competitive nature of invitations to appear on the journal cover has meant that artwork which now is appearing is no longer simply the direct output of chemistry software products (as in the examples shown above), but is generally now produced using high-level technical skills with tools like Adobe Illustrator, or 3D production software solutions such as Autodesk Maya, PovRay, 3D Studio Max, CINEMA 4D Studio (Figures 27 and 28).

Of course the expectation that a research chemist or textbook author could have the skills to use these software solutions outside their normal research computer tools is low, so the scientists have turned full circle now and are collaborating with experts in using electronic visualisation tools, such as 3D artists. One of this paper's authors, Karl Harrison, has been providing such a role for members of the Department of Chemistry at the University of Oxford (Figures 26 and 29). 
Figure 29 3D renders of chemical structures.
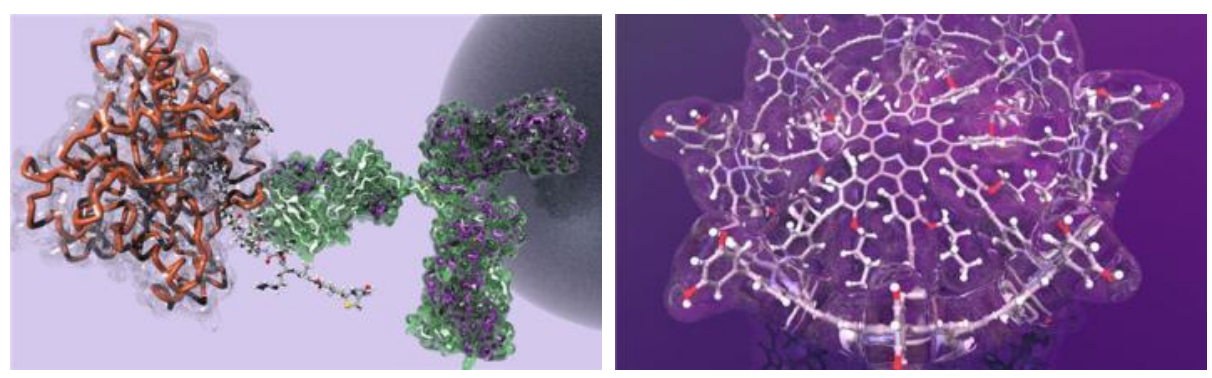

The following figures 30-35 illustrate a typical discussion and design from initial science illustration in a research paper to cover image. A research scientist at the University of Oxford had been invited to submit a cover illustration based on their novel science, which was the single step biotinylation of 5Hydroxymethylcytosine in a 100 unit single stranded DNA with N-biotinyl L-cysteine, then enrichment and isolation of biotinylated DNA using an enzyme modified nanoparticle bead Dynabeads ${ }^{\circledR}$ M-280 streptavidin. The scientist provided an initial concept for the cover art as a rough sketch (Figure 30).

Figure $30 \quad$ Initial concept sketch.

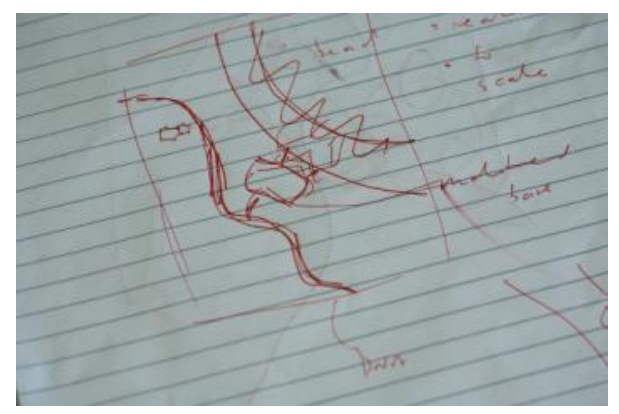

An electronic visualisation and resulting cover art therefore needed a number of components, a 3D model of the 100 unit single strand of DNA (Figure 31), modified this with the chemical addition (Figure 32), a 3D model of the protein streptavidin (Figure 33) and then an understanding of the coverage of the streptavidin protein on the nanoparticle bead.

Figure $31 \quad$ Component 1 for cover art.

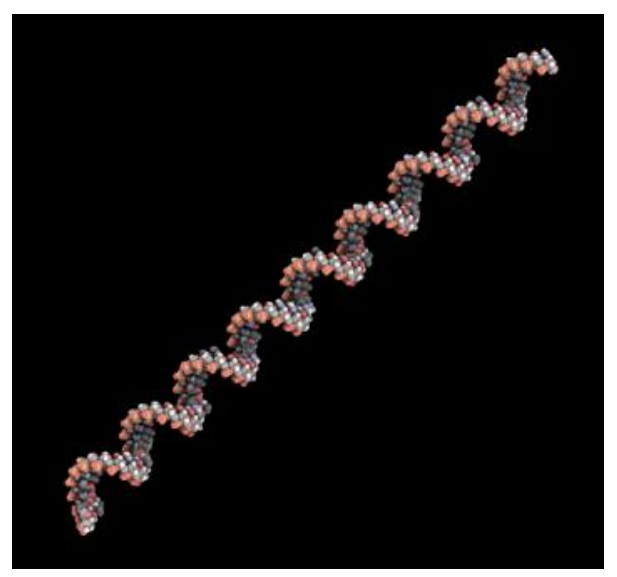


Figure 32 Component 2 for cover art.

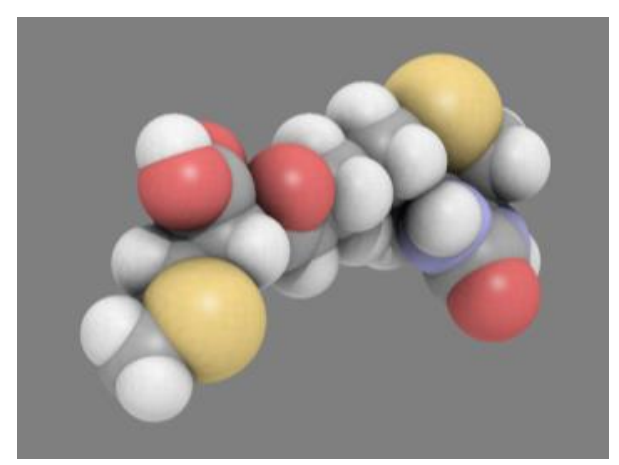

Figure $33 \quad$ Component 3 for cover art.

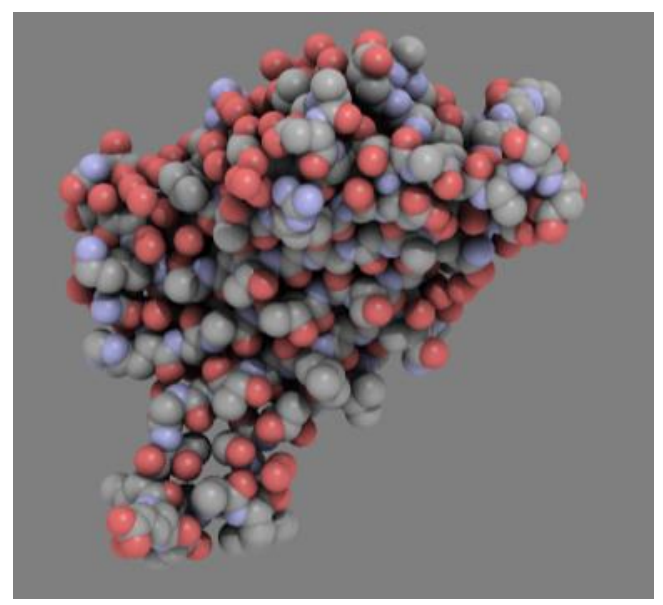

Combining all these elements together in a 3D ray-tracing program provided an initial draft shown in Figure 34 and then this was modified to build in 3D layers to produce the final illustration for the cover, which was accepted for by the journal editors (Figure 35).

Figure 34 Initial draft design.

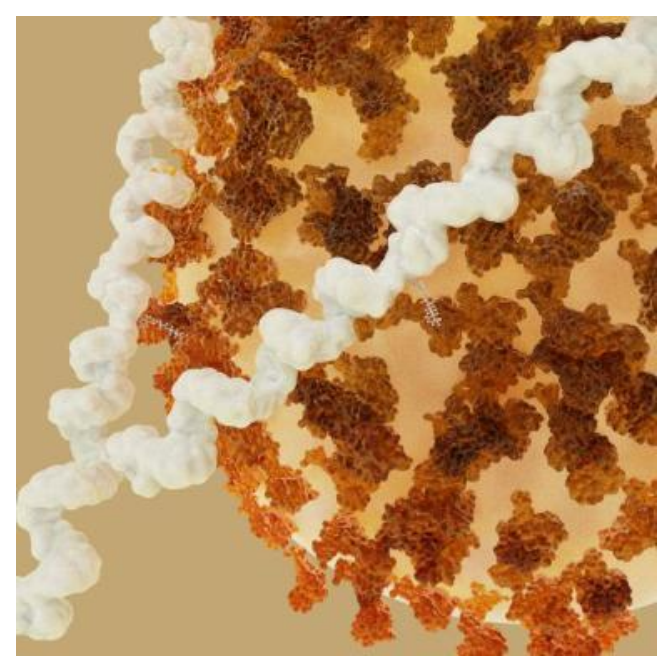


Figure 35 Cover design.

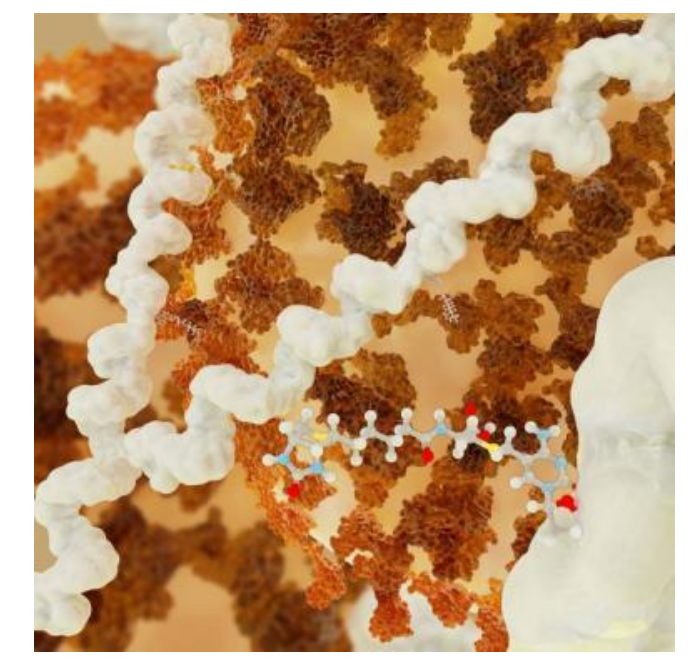

\section{Software}

The broad nature of chemistry as a modern subject, stretching from the boundaries of physics to those of molecular biology, has led to an equally broad range of tools for visualisation using molecular graphics software (Wikipedia, 2014c). It is therefore impossible to review all of the available packages used in visualisation here. Instead we hope to provide an overview of some of the wide variety of software available to chemistry research and describe some of the applications of these with respect to research. We must also draw a distinction between visualisation used during the research process and that used in a more artistic framework for the production of graphics either for journal covers or table of contents diagrams. The previous section has concentrated on the latter and therefore in this section we primarily consider visualisation as a tool for research.

\section{Visualisation as a tool for research}

In chemistry research, we can broadly divide the types of chemical systems for which electronic visualisation is frequently applied into three categories: small molecules (up to 100 atoms), large periodic systems (such as inorganic crystals) and biological systems (for example proteins and DNA). These are reflected in electronic visualisation by the file types that are used to store data for visualisation.

Within chemistry, probably the most simple file format for three-dimensional data sets is the $x y z$ format (http://openbabel.org/wiki/XYZ). These follow standard text file formats and contain information on the locations of atoms in space (Cartesian coordinates), the atom types (defined by atomic number) and the number of atoms within a system. These files do not contain any information regarding connectivity of atoms, which can be defined by the software used to visualise the system. While this file format may be suitable for small molecules, the lack of connectivity information can cause problems for larger systems.

The standard file format for biological data is .pdb files (protein databank files). As with .xyz files, these files are also text files and although based on Cartesian coordinates contain information about amino acid sequences in proteins, amino acid residue types, and atom types within each amino acid residue, which can be used to deduce connectivity information. The files also contain information about occupation of each atomic site and a temperature factor related to the localisation of the electron density corresponding to the atomic position in the original crystal structure. Within . $p d b$ files, the spacing between parameters for different types of record is very important; an explanation of this can be found online

(http://deposit.rcsb.org/adit/docs/pdb_atom_format.html).

Although the structures of many large biological molecules have been determined by X-ray crystallography, since the larger biological molecules are not found naturally as crystals in their natural environments, it is therefore not usually necessary to consider the periodicity of the crystals, although information on the crystal symmetry and periodicity is contained in the .pdb file format. (In many instances the deduction of a biological structure from a crystal can cause uncertainty over the validity of the structure found in this fashion compared to that existing in the solution state.) By contrast, for extended periodic systems such 
as ionic crystals, metal organic frameworks, zeolites, etc., it is frequently the periodicity of the structure that is of interest. It is therefore necessary to include this periodicity in any visualisation of such systems.

For periodic systems, .cif (crystallographic information file) formats are most common. This file type is promulgated by the International Union of Crystallography (IUCr) (Hall et al., 1991; Brown and McMahon, 2002). In addition to containing information about the position of a finite number of atoms (a crystal motif), .cif files also include information about the symmetry of the crystal structure that they are describing, such as the crystal cell dimensions, shape and symmetry. This allows the periodicity of the structure to be considered in any visualisation.

Furthermore, in addition to considering only the position of atoms in three-dimensional space, there are a plethora of other file types, many linked to individual programs used to simulate molecular properties such as dynamics, electronic structure, etc., which allow these additional properties to be stored and used in visualisation. Frequently it is small molecules that show the largest range of file types, primarily because the complexity of any molecular calculations increases significantly with the number of atoms considered. Thus, in the past, molecular calculations such as density functional theory were limited to small molecules. However, improvements in the calculation methodology and computational speed are allowing this to change.

The proliferation of different file types containing structural information and also the divisions between the different file types used to record this information is reflected in the range of programs used to visualise this information as many are only coded to read and write specific file types.

\section{Databases}

One of the main factors that has driven the advancement of visualisation techniques within chemistry is the availability of chemical structures found and published by one group to the wider research community. The mechanism for this distribution is via the form of online structural databases from which anyone with Internet access can download the structural files.

The Protein Data Bank (http://www.rcsb.org/pdb/home/home.do) is the depository for over 88,000 biological structures found by X-ray crystallography, NMR, or electron microscopy. For periodic systems, the Cambridge Crystallographic Data Centre (http://www.ccdc.cam.ac.uk/pages/Home.aspx) has been the primary location for depositing this type of structure. Recently, the Royal Society of Chemistry has introduced ChemSpider (http://www.chemspider.com/About.aspx). This has combined access to the Protein Data Bank, Cambridge Crystallographic Data Centre and many other structural sources into a single search engine.

Founded in 2007, Protopedia describes itself as 'The free, collaborative 3D-encyclopedia of proteins \& other molecules' (http://www.protopedia.org). This website is an interesting combination of database and structural viewer. It contains a record for all of the files in the PDB database. However, in addition to static images, it also utilises a Java-based applet to allow the user to interact with the structures directly.

\section{Programs to visualise three-dimensional structural data}

Due to the difference in the types of files used to store three-dimensional datasets for small molecule, biological, and inorganic systems, there is also somewhat of a divide in the programs used to visualise these different types of systems. However, it should be noted that many of the programs described below will operate on many different types of datasets.

\section{Small molecules}

Three-dimensional structural data for small molecules can either be found from X-ray structures or, when these are not available, approximate structures can be built using standard bond lengths and angles. These can be derived from two-dimensional data, which is converted into three-dimensional data, or built directly in three dimensions. The ChemBioOffice suite of programs allows 2D structures drawn in ChemDraw to be converted into 3D structures in ChemBio3D. Examples of programs that allow structures to be built directly in three dimensions include ChemBio3D, ChemCraft (http://www.chemcraftprog.com), and UCSF Chimera. Whereas ChemBio3D and ChemCraft allow the molecule to be built by dragging components into position using a mouse, Chimera has the problem that molecules can only be built an atom at a time, by adding atoms to or modifying pre-existing atoms, which can make construction of molecules using this software time-consuming. Many quantum chemical calculation or density functional theory (DFT) programs such as Amsterdam Density Functional (ADF, http://www.scm.com) and Gaussian (http://www.gaussian.com/g tech/g ur/k dft.htm), both commercial pieces of software, have inbuilt graphical user interfaces that allow molecules to be created in three 
dimensions. ChemCraft, Gaussian, and ADF also allow molecules to be altered to conform to a specific symmetry or space group, which is an important feature when defining structures for use in calculations, since the presence of symmetry can reduce calculation times significantly.

\section{Periodic systems}

The symmetry of periodic lattice systems means that programs used to visualise this type of structure have tools to allow the symmetry of the system to be defined and analysed. In addition to the atoms in the crystals molecular structure and the bonding between them, it can also be the space between the atoms in the crystal structure that is of interest. Therefore, software may also allow analysis of these voids. As seen in Figure 22, there are many different ways of representing crystal structures, not all of which show the atoms in the crystal explicitly.

Two of the leading programs for visualizing this type of system are Mercury (http://www.ccdc.cam.ac.uk/Solutions/CSDSystem/Pages/Mercury.aspx), which is free to download, and Crystal Maker Suite (http://crystalmaker.com/crystalmaker/index.html), which has a free demonstration version. The analysis of crystallographic data is by its visualisation as it is used to build a structure,. For this reason even programs used to analysis raw crystallographic data frequently have inbuilt visualisation functions. An example of this is the freely downloadable program Crystals (http://www.xtl.ox.ac.uk/crystals.html).

\section{Biological molecules}

There are a myriad of programs that are available to view .pdb files (including UCSF Chimera, VMD, Swiss PDB viewer, Pymol, and YASARA - Yet Another Scientific Artificial Reality Application). A reasonably comprehensive list of available programs is given on the Research Collaboratory for Structural Bioinformatics (RCSB) Protein Data Bank (PDB) website (http://www.rcsb.org/pdb/static.do?p=software/software_links/ molecular graphics.html). Among those that are freely available to download, the most widely used are VMD, Pymol, and Chimera (see results from a survey on the Rosetta molecular modelling blog http://rosettadesigngroup.com/blog/284/what-is-your-favorite-molecular-viewer/). All read a large variety of file types and render images of proteins in various different representations, including full atomic representations such as wire, ball and stick, space filling, and also backbone-only representations such as ribbons. Surfaces such as solvent accessible surfaces of biomolecules can also be generated in most programs. Of these programs, UCSF Chimera is probably the most advanced in terms of its visual rendering of images within the program, allowing the user either to select visualisations preset to provide good quality images for publication or to alter visualisation properties individually, such as the colour, depth cue, and lighting of molecules (see Figure 36). 
Creative Visualisation in Chemistry

\section{J. P. Bowen, A. M. Bowen, and K. Harrison}

Figure 16 Screen captures of Chimera showing some of the functionality from the menus. Image 1: Lighting control. Image 2: Depth of viewing control. Image 3: Colour selection. Image 4: Preset option for publication quality images.

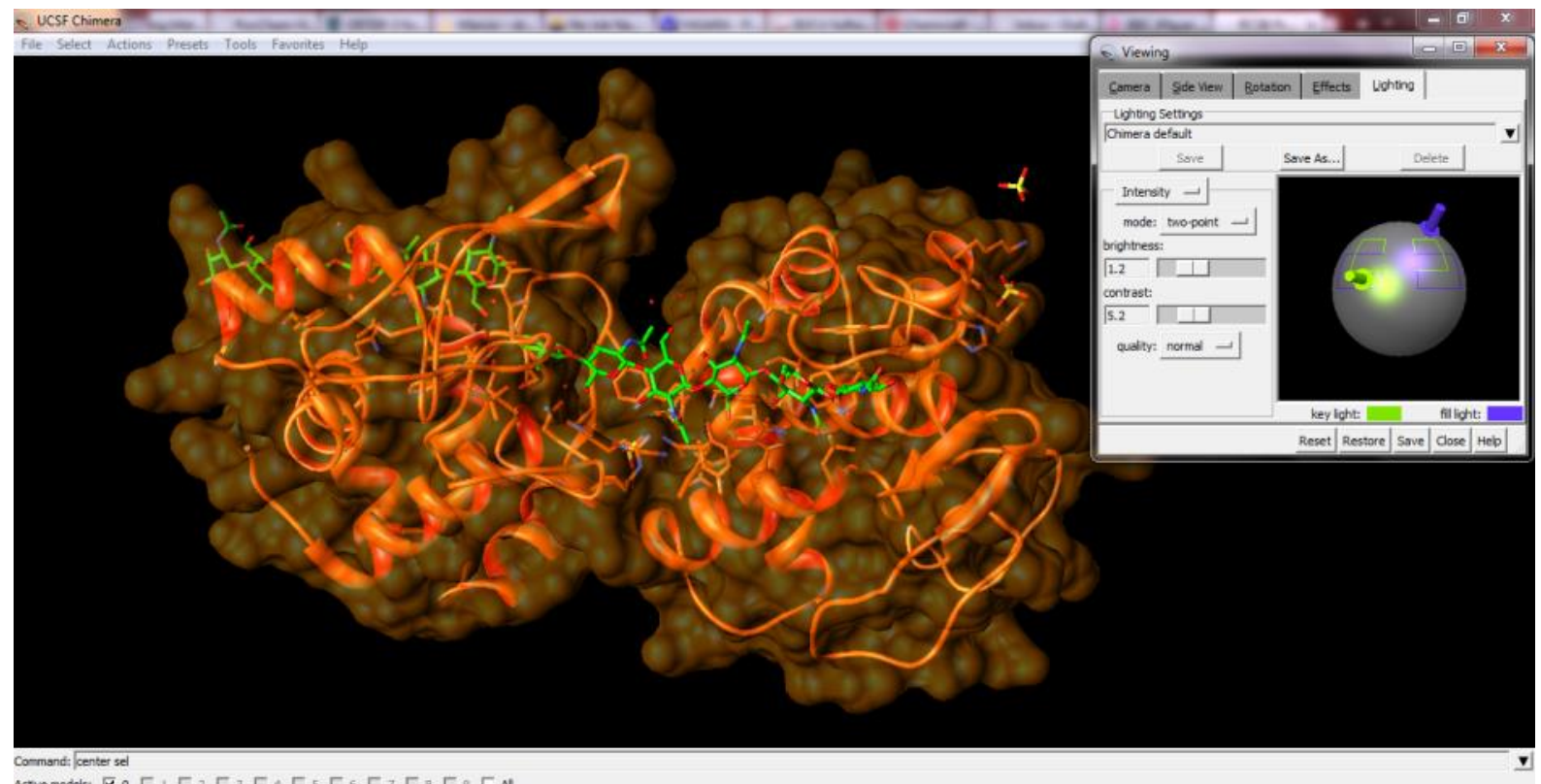

Commend: center sel

acove models: $\nabla 0 \Gamma_{1} \Gamma_{2} \Gamma_{3} \Gamma_{4} \Gamma_{5} \Gamma_{6} \Gamma_{7} \Gamma_{\theta} \Gamma_{9} \Gamma_{A}$
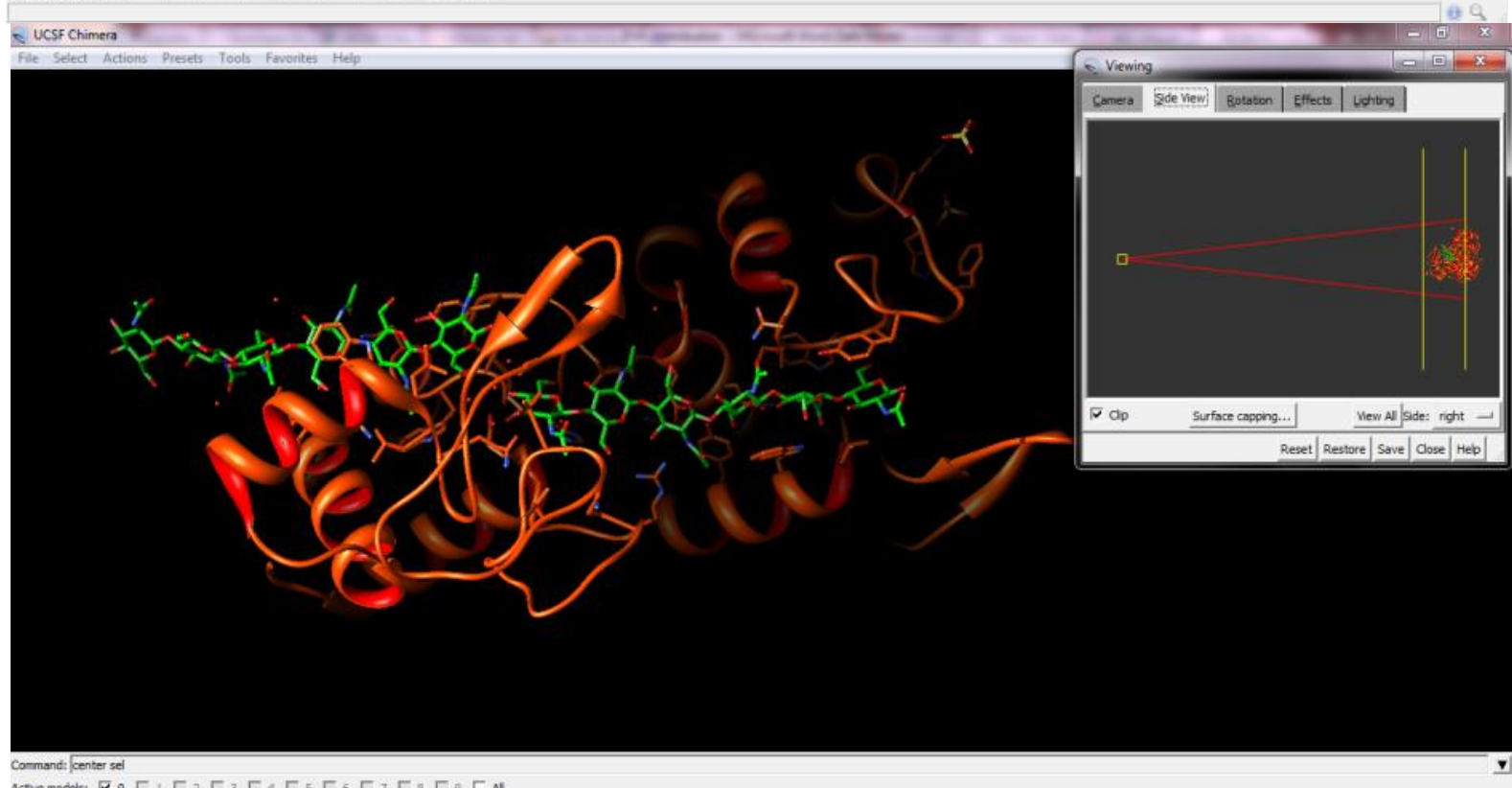

Acove models: $\nabla_{0} \Gamma_{1} \Gamma_{2} \Gamma_{3} \Gamma_{4} \Gamma_{5} \Gamma_{6} \Gamma_{7} \Gamma_{4} \Gamma_{0} \Gamma_{\mathrm{A}}$ 

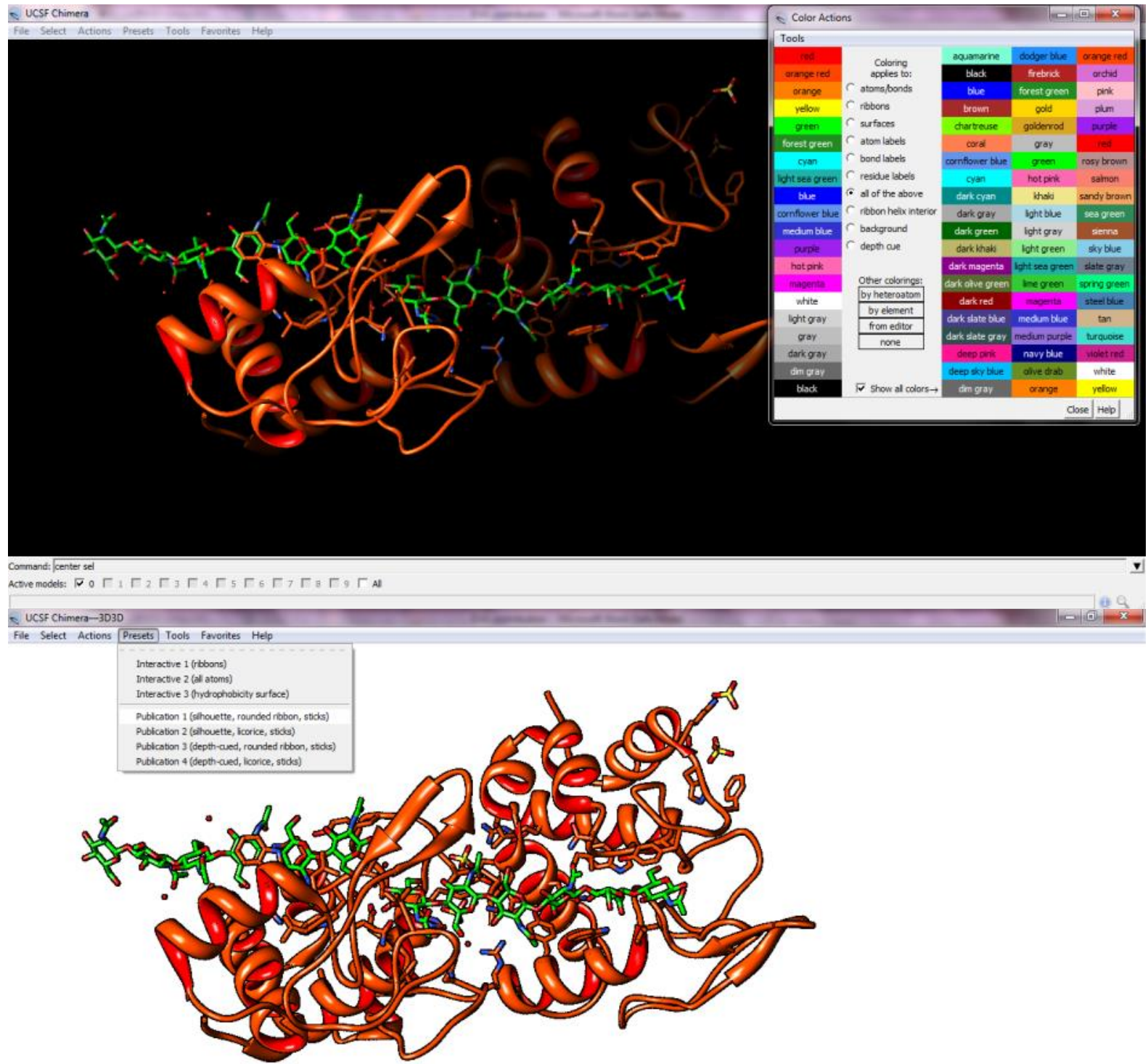

acovere models : $\nabla_{0} \Gamma_{1} \Gamma_{2} \Gamma_{3} \Gamma_{4} \Gamma_{5} \Gamma_{6} \Gamma_{7} \Gamma_{8} \Gamma_{9} \Gamma_{\text {Al }}$

Surface 3d3d, category ligand has 1 components

Since the nature of biological molecules is usually complex due to their size, it can be helpful in some cases to consider only a small region of the molecule, such as the active site in an enzyme. LigPlot (Wallace, 1996) and LigPlot+ (Laskowski, 2011) produce a two-dimensional visualisation of a ligand bound within a protein or a protein-protein interface. In this visualisation (see Figure 37), hydrogen bonds are displayed explicitly as green lines and hydrophobic contacts are represented by fringed arcs, somewhat akin to the symbols used on maps to represent viewpoints. Any protein structure not forming part of the interaction interface is removed, thus truncating the protein structure. LigPlot ${ }^{+}$can be linked into either PyMol or RasMol in order to visualise the truncated two-dimensional structure in three dimensions. Also the screen can be split into two panels to allow comparison of two structures. One limitation of these programs is that the only output file type for the two dimensional image files is PostScript format; this can prove problematic for scientists writing using LaTeX and compiling as PDF format. 
Figure 37 Output image from LigPlot ${ }^{+}$for one of the sugar chains bound to lambda phage lysozyme in the $p d b$ structure $3 d 3 d$ (Leung, 2001).

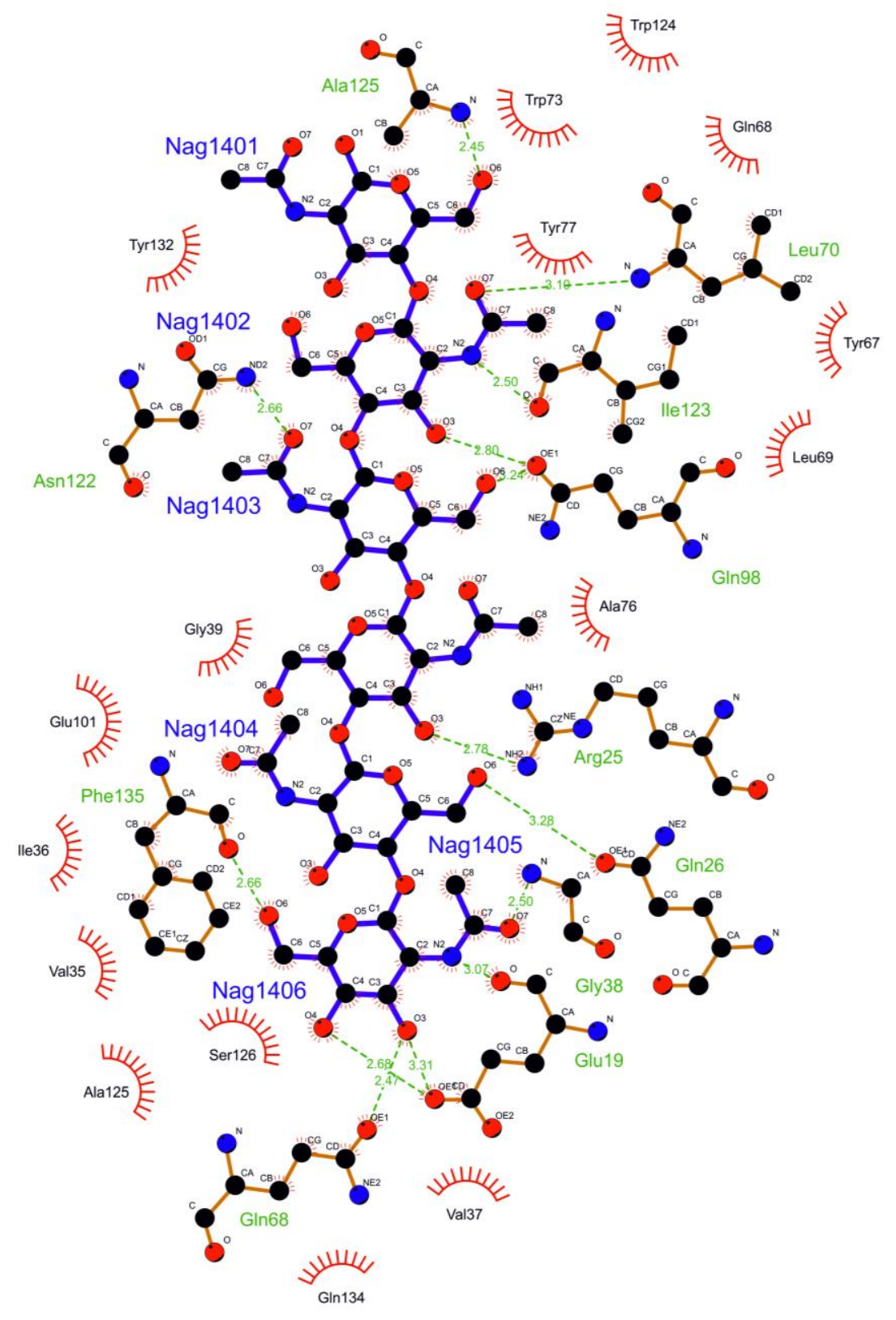

$3 d 3 d$

\section{Specialist software packages}

Research into molecules is by its very nature a specialist field. Therefore, over the last few decades as many researchers have become literate in programming languages, they have written software packages to enable them to progress their own research. This expansion has necessarily paralleled the rise of computational chemistry as a field in its own right.

Since the nature of scientific research is generally collaborative, many of these programs are now available for scientists to download freely from the Internet and to use for their own research. The number of these packages is large and therefore here we highlight a few that are used specifically to visualise molecular motion and properties which are not specifically structural. This covers any information that is not directly static molecular coordinates, which can be viewed relative to an atomic structure. 


\section{Visualizing motion and conformational flexibility}

It must be remembered that all chemical and biochemical systems are in fact dynamic and therefore the depiction of motion within molecular structures is of vital importance. With the rapid development of electronic visualisation, frequently the most convenient method for this is via animations, which can show the motion of a system with respect to time. Although these have become somewhat de rigueur for computer-based presentations, alternative methods of visualizing motion are still required for printed resources.

\section{Superimposing snapshots}

One straightforward method for illustrating a molecular dynamics trajectory is to take static snapshots of the molecule at different points and overlay these. In order to obtain a good overlay, it is necessary to minimise the change in overall orientation of the molecule. This can be done by matching the structures using a least squares type algorithm to minimise the separation between the atomic coordinates. An example of this is shown in Figure 38.

Figure 38 Overlay of structural files from a molecular dynamics simulation in GROMOS (http://www.gromos.net), each structure occurring at nanosecond intervals in the trajectory of the simulation of molecule A from crystal structure 1 AM7 at 300Kelvin (Smith, 2013). The colours follow a rainbow pattern where 0ns is red and 10ns is white. The image was produced using Chimera (Pettersen, 2004) and the structures matched together using the "matchmaker" facility, which minimises the separation between the protein backbone of different structures.

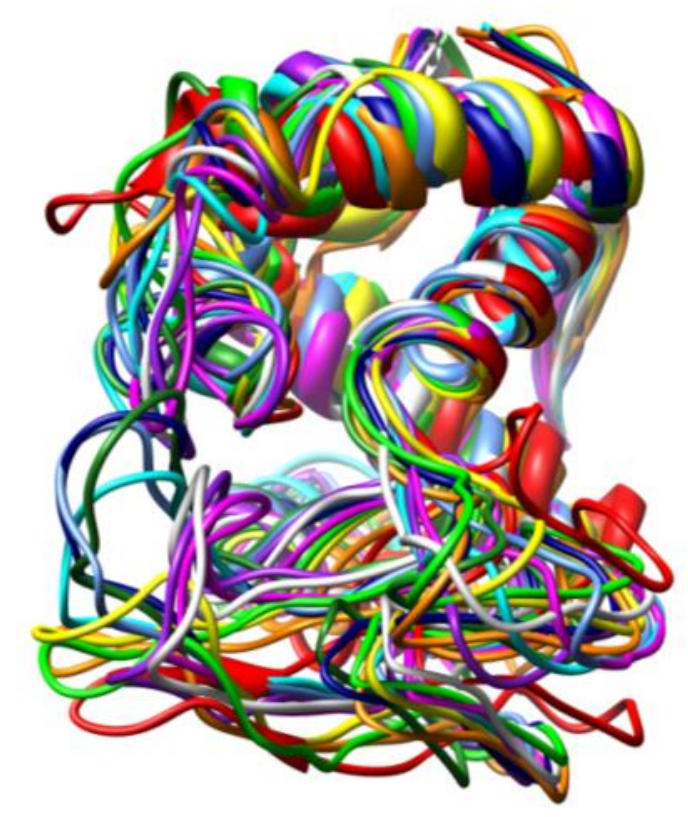

Alternatively, when the motion of the protein is large, for example corresponding to the movement of a whole domain, such that it can be modelled satisfactorily by only two structures, it is possible to compare these two structures and to draw a vector representing the motion of each section of the molecule. An example is shown in Figure 39; one structure is displayed as a backbone coil and the relative position of the second is demonstrated by conical arrows. 
Figure 39 Image taken from Front cover of MMM 2011 user manual

(http://www.epr.ethz.ch/software/MMM 2011.2 manual.pdf). The picture shows the structural change of maltose binding protein MalE from the open apo form (PDB 1OMP pale green coil model) to the closed substrate binding form (PDB $3 \mathrm{MBP})$. The new motion command was used to generate this plot.

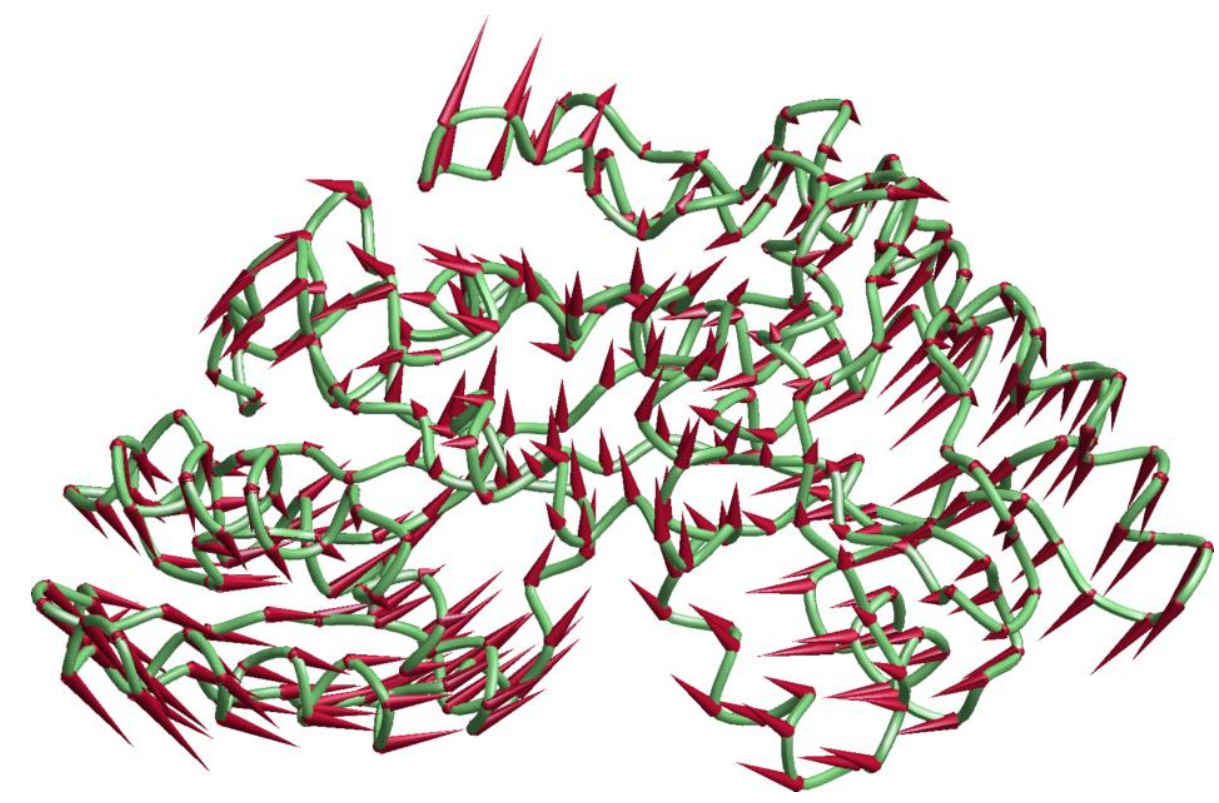

Sometimes researchers are only interested in the motion of small part of a molecule. For example, synthetic labels are often added to proteins for use in spectroscopy such as Electron Spin Resonance (ESR) and Forster Resonance Electron Transfer (FRET). Frequently the dynamics of these labels relative to the protein backbone affect the spectroscopic results and therefore it is important to understand the motion of these labels, which is fast relative to the protein backbone and therefore it is possible to consider this motion relative to a static protein backbone. The position of the label relative to the protein backbone can be displayed as a distribution of "conformers" (similar molecules differing by the rotation of a single bond). Figure 40 was generated using MMM (http://www.epr.ethz.ch/software); this models the position of a nitroxide spin label attached to a protein using a pre-computed library of nitroxide rotamers (sets of conformers). The occupancy of each rotamer conformation is evaluated and an image is produced showing the protein structure with a distribution of the oxygen atoms (magenta spheres) of occupied rotamer conformers relative to the protein structure. The size of the sphere is related to the occupation of the confirmation it represents.

Figure $40 \quad$ Left: The structure of the MTSL spin label, plotted in ChemDraw) with the flexible bonds marked. The sulphide (S-S) bond can adopt either a cis- or a trans- conformation. However, due to the relatively high polarisability of the sulphur electron density, there is partial double bond character in the S-S bond. Therefore, the cis- and trans- forms of the bond are predominant over any intermediate angles and interconversion between the two forms is slow. Right: A possible distribution of nitroxide positions calculated for an MTSL-labelled ferredoxin protein (3HUI.pdb) (Bell et al., 2010) calculated using MMM.

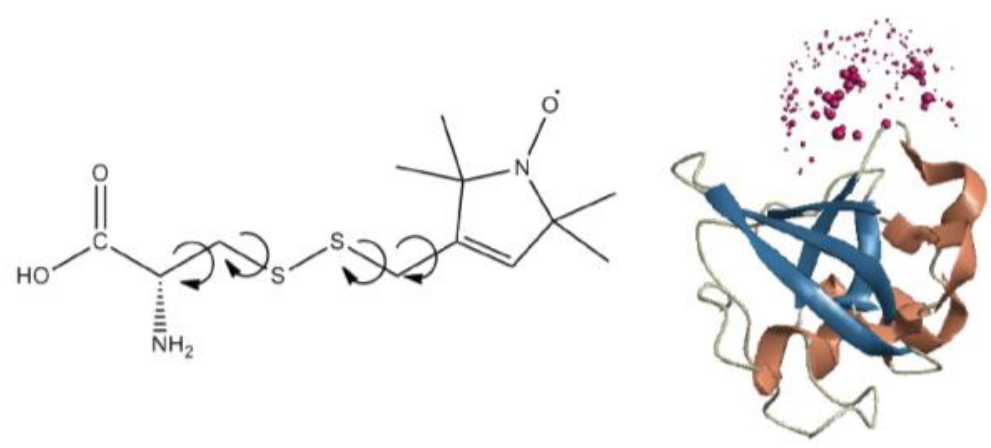

Thermal ellipsoids from crystallography 
Thermal ellipsoids, more formally termed atomic displacement parameters, are parameters used in crystallography to indicate the magnitudes and directions of the thermal vibration of atoms in a crystal structure. Two pieces of software used to plot these ellipsoids are Ortep (http://www.chem.gla.ac.uk/ louis/software/ortep3/) and Gretep (http://www.ccp14.ac.uk/tutorial/lmgp/gretep.html). Both are freely available to download. An example is shown in Figure 41.

Figure $41 \quad$ Thermal ellipsoids plotted in Ortep

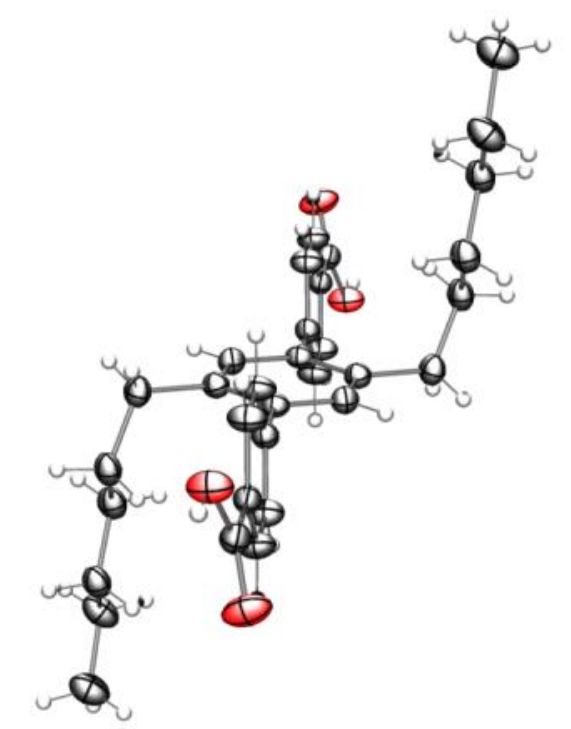

\section{Visualizing non-structural information with respect to a molecular structure}

Frequently in molecular research, it is convenient to depict non-structural data relative to a molecular structure to which it is related.

\section{Electron density maps}

Electron density maps are three-dimensional descriptions of the electron density in a crystal structure. They are determined from X-ray diffraction experiments and the resulting X-ray crystal structure is modelled to match the electron density map. They can be evaluated as parallel two-dimensional contoured sections at different heights in the crystallographic unit cell or as a three dimensional plot. Before the use of computers, threedimensional electron density maps were made out of layers of Perspex fitted together with the density drawn on each layer (see Figure 42). 
Figure 42a Electron density map of potassium benzyl penicillin and the skeletal representation of the penicillin molecule. Derived by Dorothy Hodgkin from the X-ray diffraction pattern of penicillin crystals (http://www.portlandpress.com/pp/books/online/tiepac/session6/ch2.htm).
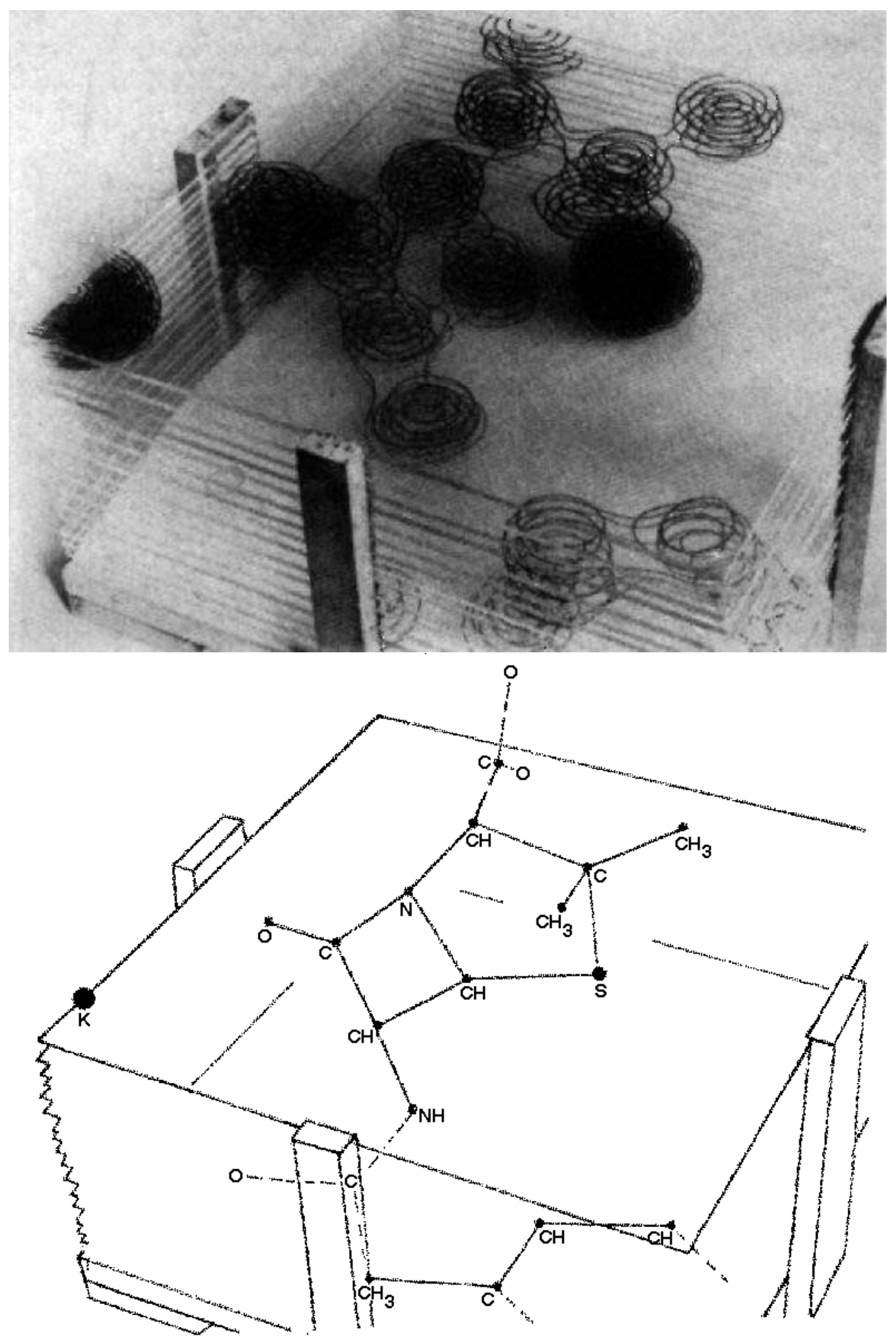
Figure 42b A modern electron density map, Snapshot of $1.0 \AA$ A electron density map at sigma level 1, displayed in Jmol (http://www.proteopedia.org/wiki/index.php/Electron_density_maps).

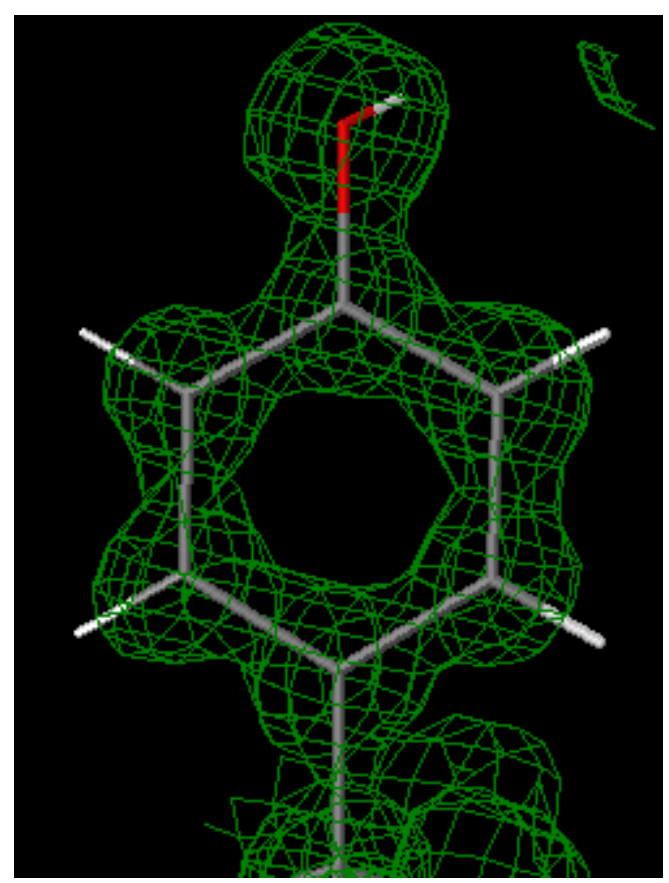

Nowadays, an electronic density map is much more easily visualised using software such as Jmol, an opensource Java viewer for chemical structures in 3D (http://www.jmol.org). An online demonstration of this feature is available (http://www.umass.edu/molvis/edm/cloud/).

\section{Molecular orbitals from DFT}

Density Functional Theory (DFT) is a quantum mechanical modelling method, based on atomic positions of a molecule the electron energy levels and orbital shapes can be calculated. Most DFT software provides users with a GUI in which to view the calculated orbitals, as shown in Figure 43. 
Figure 43 Pictorial representation of the highest occupied molecular orbital of the copper porphyrin fragment, calculated using DFT (ADF tool) and plotted using ADF view. This shows that a large degree of the electron density (coloured red and blue for the positive and negative portions) of this orbital containing the unpaired electron is delocalised onto the nitrogen ligating the copper centre (the pink sphere).

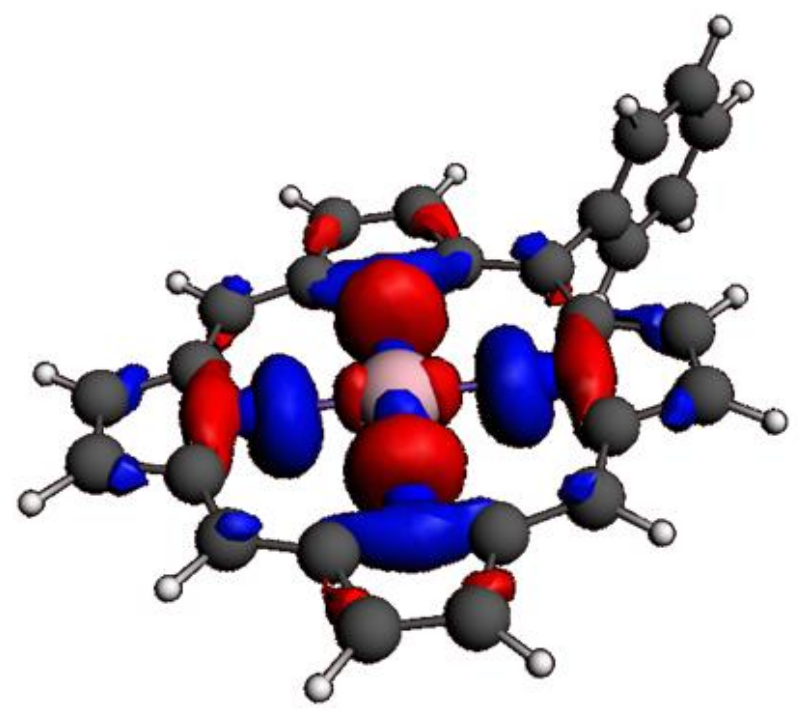

\section{Coloured solvent accessible surfaces}

The solvent accessible surface of a molecule is calculated by rolling a ball with a diameter equivalent to a water molecule around the molecule and by plotting the surface formed by the centre of the sphere. This surface is frequently coloured to allow the visualisation of molecular properties such as columbic or electrostatic charges or spectroscopically derived data such as NMR line broadening behaviour, which can be used to show mobile regions of the molecule.

Figure 44 The solvent accessible surface of Lambdaphage Lysozyme; 3d3d (Leung, 2001), coloured with columbic charges. The figure was produced using Chimera.

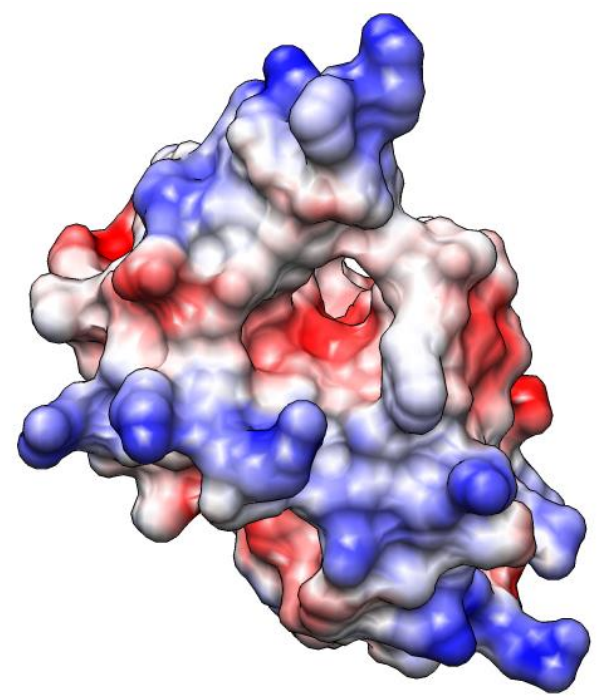




\section{Hyperfine tensors and g-tensors}

Hyperfine tensors quantify the coupling of an unpaired electron to a nucleus and g-tensors quantify the resonance field of the unpaired electron. These can be calculated by DFT or extracted directly from experiment. Figure 45 shows the output of a Matlab program written by a physical chemistry researcher to plot these tensors (Abdalla, 2012). Spinach, a spin dynamics simulation package also based on Matlab, is free to download and can also be used to plot this type of tensor (http://spindynamics.org/Spinach.php). (Hogben, 2011)

Figure 45 The hyperfine tensors of all protons included in the model plotted on the molecular structure. The isotropic part has been scaled by $1 / 100$ to show the anisotropy of the hyperfine tensors more clearly.

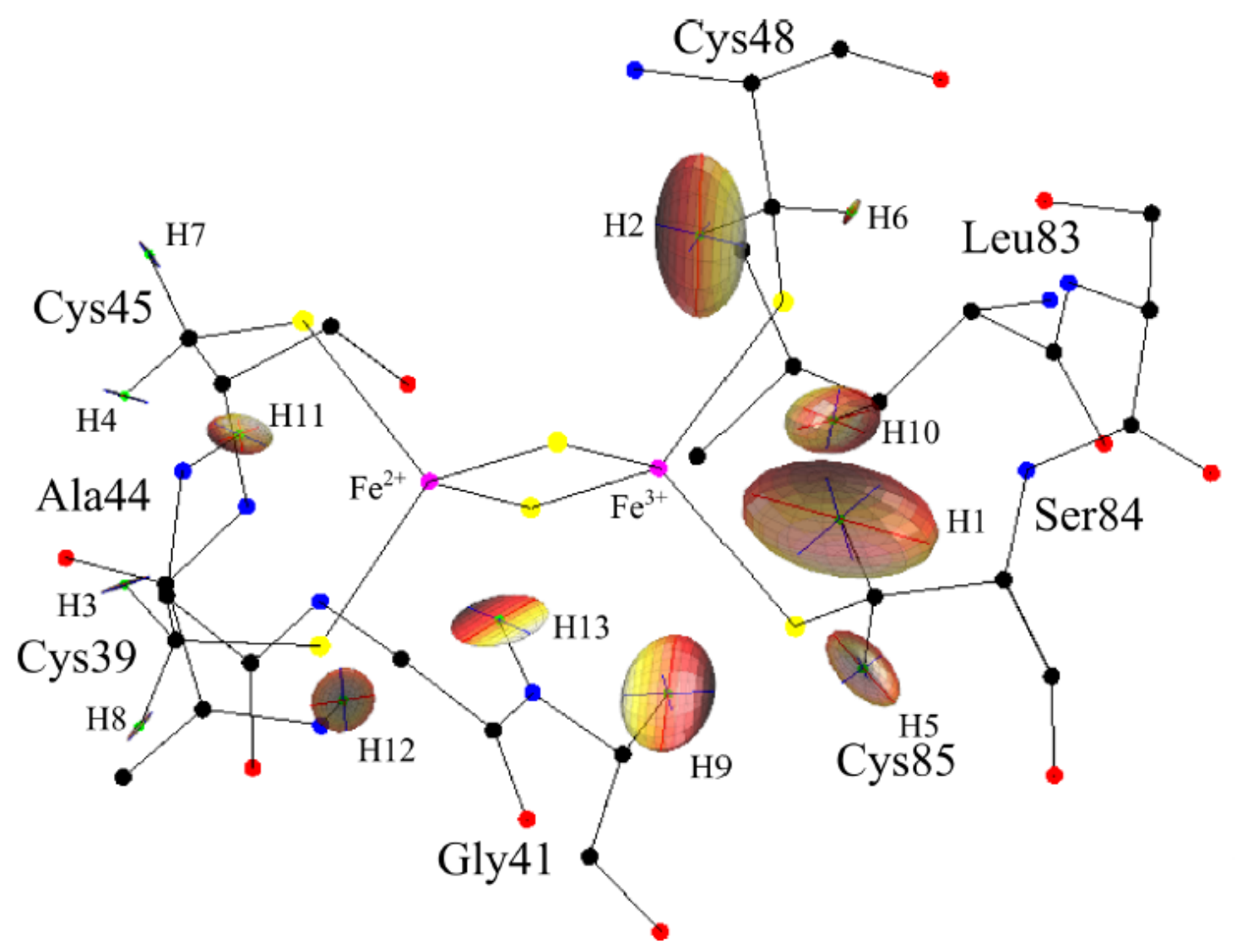


Figure 46 Screen shot of Spinach GUI taken from the web (http://spindynamics.org/Spinach-GUI.php) showing hyperfine tensors relative to a molecular structure.

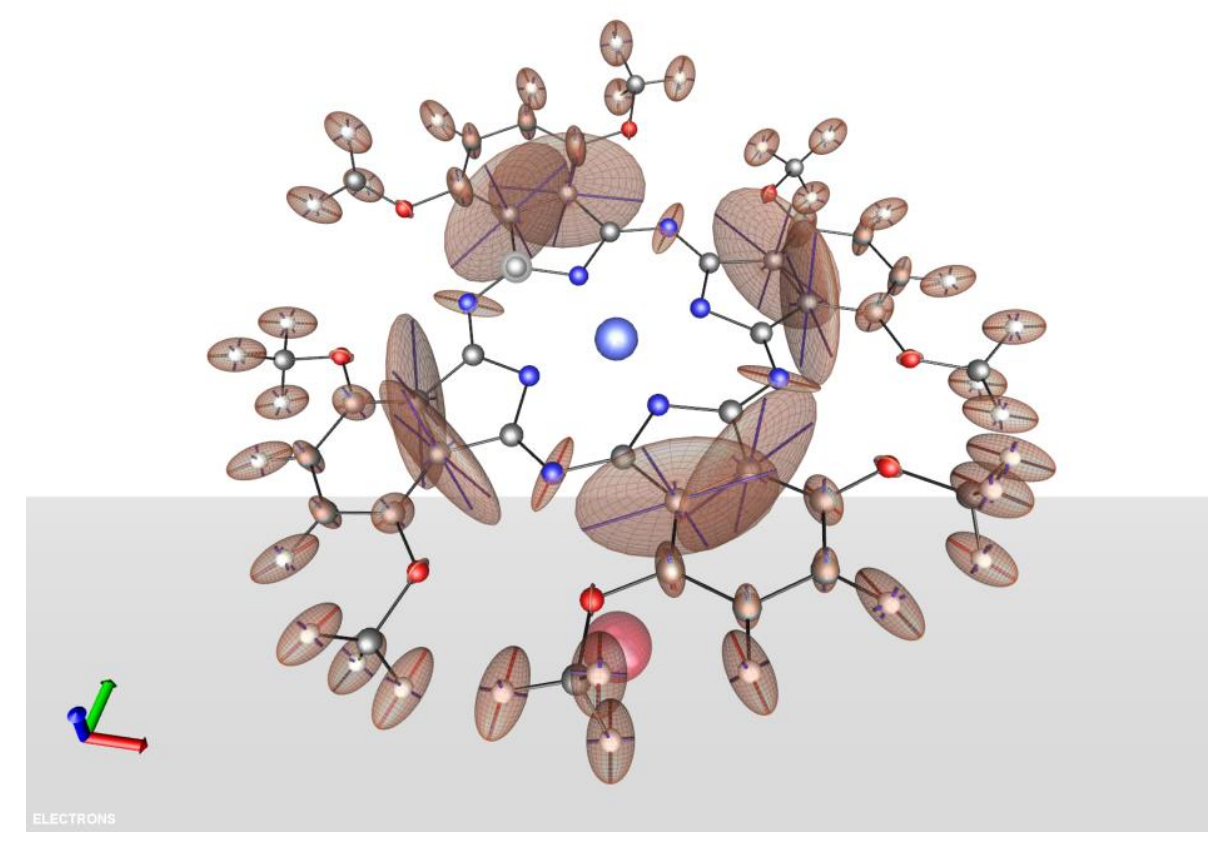

\section{Summary}

We have presented some selected software to aid in molecular visualisation. Much further specialist software has been produced, such as Gabedit (Allouche, 2011), Molden (Schaftenaar \& Noordik, 2000), Molekel (Portmann \& Lüthi, 2000), etc. For a more extensive selection of chemistry visualisation software, especially for those interested in the possibility of using such software for artistic purposes, Wikipedia provides a list of molecular graphics software systems (Wikipedia, 2014b).

\section{Conclusion}

This paper has presented the visualisation of molecules using software for artistic and scientific purposes. The move of molecular graphics from scientific use towards artistic use has been considered (Atwood et al., 2003). The combinations of facilities for handling 3D meshes, materials, instancing, depth of field, reflection mapping, High-Dynamic Range Imaging (HDRI) and Image Based Lighting (IBL), which are available in 3D rendering software solutions, can provide highly abstract or apparently photorealistic illustrations. This has led to an exciting time where scientists are working with artists using creative visualisation to view chemistry in new ways and also to show the public the beauty in the science they are undertaking (Phipps, 2008).

There is a wide variety of software used for molecular visualisation. The structure of these molecules can be very complex, such as in the case of enzymes, proteins, etc., and even illustrations for scientific use can have artistic aspects for a non-specialist viewer; e.g., see Bowen (2008; 2013a) and Lovett et al. (2009), which inspired the origins of this current paper (Harrison et al., 2013).

In summary, this paper illustrates the aesthetic possibilities of visualisation in chemistry (Hoffmann, 2003), especially at the molecular level, the historical development of illustrating molecules, and the current state of the art in this area. It is hoped that this may inspire digital artists to undertake future interdisciplinary collaboration with chemists from a more purely artistic standpoint and to utilise the wide selection of available visualisation software, as used by chemists in their scientific endeavour, for artistic purposes.

This paper has concentrated on static images. Of course, molecules can also move, if large in interesting ways. For the future, animation of molecular reconstructions is becoming increasingly possible and important (Physics arXiv Blog, 2013). Some tools towards visualisation of dynamic aspects have been covered 
in this paper. Biological microorganisms such as bacteria can also be visualised effectively with similarly artistic results (Kjærgaard \& Andersen, 2014). The ever-greater pervasive use of digital technology (Bowen \& Giannini, 2014) means that even a relatively specialist area such as molecular visualisation may become more ubiquitous, with visualisation tools available on smart mobile devices.

\section{Acknowledgements}

Jonathan Bowen is grateful to Museophile Limited for financial support. A part of this paper was previously presented at the EVA London 2013 Conference on Electronic Visualisation and the Arts (Harrison et al., 2013).

\section{References}

Abdalla, J.A.B., Bowen, A.M., Bell, S.G., et al. (2012) 'Characterisation of the paramagnetic [2Fe-2S] centre in Palustrisredoxin-B (PuxB) from Rhodopseudomonas palustris CGA009: magnetic moment and g-matrix determination', Physical Chemistry Chemical Physics, Vol. 14, pp. 6526-6537.

Alcorn, P.A. (1997) 'Creativity and innovation: the critical link', in Social Issues in Technology: A Format for Investigation, $2^{\text {nd }}$ edition, Prentice Hall, Upper Sadler River, Chapter 3, pp. 41-58.

Allouche, A.-R. (2011) 'Gabedit-A graphical user interface for computational chemistry softwares', Journal of Computational Chemistry, Vol. 32, No. 1, pp. 174-182. DOI: $10.1002 /$ jcc.21600

Atkins, P.W. (2003) Atkins' Molecules, $2^{\text {nd }}$ edition, Cambridge University Press, Cambridge.

Atwood, J.L. Leonard, J. and Barbour, L.J. (2003) Molecular graphics: from science to art, Crystal Growth \& Design, Vol. 3, No. 1, pp. 3-8. DOI: $10.1021 / \mathrm{cg} 020063 \mathrm{O}$

Bakshee, I. (1999) The Pattern of Beauty: The Art of Igor Bakshee, Wolfram Media, Graphica 2: The World of Mathematica Graphics.

Bell, S.G., Xu, F., Johnson, E.O.D., Forward, I.M., Bartlam, M., Rao, Z., and Wong, L.-L. (2010) Journal of Biological Inorganic Chemistry, Vol. 15, pp. 315-328.

Bowen, A.M. (2008) Studies of Bacteriophage Lambda Lysozyme, MChem thesis, Department of Chemistry, University of Oxford, UK.

Bowen, A.M. (2013a) Magnetic Resonance Studies of Proteins and Model Systems, DPhil thesis, Department of Chemistry, University of Oxford, UK.

Bowen, E.J. (1946) The Chemical Aspects of Light, $2^{\text {nd }}$ edition, Oxford University Press, Oxford, p. 223.

Bowen, H.J.M. (1966) Trace Elements in Biochemistry, Academic Press, London, p. 133.

Bowen, J.P. (2013b) 'Online communities: visualization and formalization', in Cyberpatterns 2013: Proc. Second International Workshop on Cyberpatterns - Unifying Design Patterns with Security, Attack and Forensic Patterns, Abingdon, UK, 8-9 July 2013, Oxford Brookes University.

Bowen, J.P. and Borda, A. (2009) 'Communicating the public understanding of science: The Royal Society website'. International Journal of Technology Management, Vol. 46, No. 1/2, pp.146-164.

Bowen, J.P. and Giannini, T. (2014) 'Digitalism: the new realism?', in K. Ng, J.P. Bowen, and S. McDaid (Eds.), Proceedings of EVA London 2014: Electronic Visualisation and the Arts, British Computer Society, Electronic Workshops in Computing, pp. 324-331. DOI: 10.14236/ewic/eva2014.38

Bowen, J.P. and Giannini, T. (2015) 'Galois connections: Mathematics, art, and archives', in K. Ng, J.P. Bowen, and N. Lambert (Eds.), Proceedings of EVA London 2015: Electronic Visualisation and the Arts, British Computer Society, Electronic Workshops in Computing, pp. 176-183. DOI: 10.14236/ewic/eva2015.18

Bowen, J.P. and d'Inverno, M. (2006) 'Confessions of computer scientists working in the arts: a tale of two techies', in J. Hemsley, S. Keene, L. MacDonald, J.P. Bowen, V. Cappellini, and G. Stanke (Eds.), EVA 2006 London Conference Proceedings, London, UK, 26-28 July 2006. EVA Conferences International, pp. 15.1-15.10.

Bowen, J.P., Keene, S. and Ng, K. (Eds.), (2013) Electronic Visualisation in Arts and Culture, Springer, London, Series on Cultural Computing.

Brown, I.D. and McMahon, B. (2002). 'CIF: the computer language of crystallography', Acta Crystallographica B, Vol. 58 (Pt 3 Pt 1), pp. 317-324. PMID 12037350.

Brown, P., Gere, C., Lambert, N. and Mason, C. (2008) White Heat Cold Logic: British Computer Art 1960-1980, The MIT Press, Cambridge MA.

Darius, J. (1984) Beyond Vision, Oxford University Press, Oxford.

Dickerson R.E. and Geis, I. (1976) Chemistry Matter and the Universe: Integrated approach to general chemistry, Benjamin-Cummings Publishing Co., Addison Wesley Longman, USA. 
Edmonds, E. and Franco, F. (2013) 'From communications game to Cities Tango', International Journal of Creative Computing, Vol. 1, No. 1, pp. 120-132.

Edmonds, E. and Quantrill, M. (1999) 'An approach to creativity and process', in R. Ascott (ed.), Reframing Consciousness, Intellect Books, Exeter, UK, pp.257-261, part 3, Art, chapter 9, Creative Process.

Fleming, I. (1967) 'Absolute configuration and the structure of Chlorophyll', Nature, Vol. 216, pp. 151-152. DOI: $10.1038 / 216151 \mathrm{a} 0$

Gayford, M. (2011) A Bigger Message: Conversations with David Hockney, Thames \& Hudson.

Goodwin T.W. (1964) Structure and Activity of Enzymes, Academic Press, London.

Greene, R. (2004) Internet Art, Thames \& Hudson, London, World of Art.

Grundon, M., (1962) Organic Chemistry: An introduction, Oldbourne, London.

Hall, S.R., Allen F.H. and Brown I.D. (1991) 'The Crystallographic Information File (CIF): a new standard archive file for crystallography'. Acta Crystallographica A, Vol. 47, No. 6, pp. 655-685. DOI: 10.1107/S010876739101067X

Harrison, K., Bowen, J.P. and Bowen, A.M. (2013) 'Electronic visualisation in chemistry: from alchemy to art', in K. Ng, J.P. Bowen, and S. McDaid (Eds.), Proceedings of EVA London 2013: Electronic Visualisation and the Arts, British Computer Society, Electronic Workshops in Computing, pp. 267-274.

Hoffmann, R. (2003) 'Thoughts on aesthetics and visualization in chemistry', HYLE - International Journal for Philosophy of Chemistry, Vol. 9, No. 1, pp. 7-10.

Hogben, H.J., Krzystyniak, M., Charnock, G.T.P., Hore, P.J., and Kuprov, Ilya (2011) 'Spinach - a software library for simulation of spin dynamics in large spin system', Journal of Magnetic Resonance, Vol.. 208, No. 2, pp. 179194. DOI: $10.1016 /$ j.jmr.2010.11.008

Hugill, A. and Yang, H. (2013) 'The creative turn: new challenges for computing', International Journal of Creative Computing, Vol. 1, No. 1, pp. 4-19.

IUPAC (1997) Compendium of Chemical Terminology, $2^{\text {nd }}$ edition (the "Gold Book"). A. D. McNaught and A. Wilkinson (Eds.). Blackwell Scientific Publications, Oxford. M. Nic, J. Jirat, and B. Kosat (Eds.) (2006-), Gold Book. URL: http://goldbook.iupac.org (accessed 24 August 2014). DOI: 10.1351/goldbook

Jones, L.L., Jordan, K.D. and Stillings, N.A. (2005) Molecular visualization in chemistry education: the role of multidisciplinary collaboration, Chemistry Education Research and Practice, Vol. 6, pp. 136-149. DOI: 10.1039/B5RP90005K

Kjærgaard, R.S. and Andersen, E.S. (2014), Seeing Streptococcus pneumoniae, a common killer bacteria, in K. Ng, J.P. Bowen, and S. McDaid (Eds.), Proceedings of EVA London 2014: Electronic Visualisation and the Arts, British Computer Society, Electronic Workshops in Computing.

Laskowski R.A. and Swindells M.B. (2011) 'LigPlot+: multiple ligand-protein interaction diagrams for drug discovery', Journal of Chemical Information and Modeling, Vol. 51, pp. 2778-2786. PubMed id: 21919503

Laszlo, P. (2003) 'Foundations of chemical aesthetics', HYLE - International Journal for Philosophy of Chemistry, Vol. 9, No. 1, pp. 11-32.

Liu, Z., Yan, H., Wang, K., Kuang, T., Zhang, J., Gui, L., An, X., and Chang, W. (2004) 'Crystal structure of spinach major light-harvesting complex at 2.72 A resolution', Nature, Vol. 428, pp. 287-292. PubMed: 15029188. DOI: 10.1038 /nature 02373

Lovett, J.E., Bowen, A.M., Timmel, C.R., Jones , M.W., Dilworth, J.R., Caprotti, D., Bell , S.G., Wong, L.L. and Harmer, J. (2009) 'Structural information from orientationally selective DEER spectroscopy', Physical Chemistry Chemical Physics, Vol. 11, pp. 6840-6848. DOI: 10.1039/B907010A

Leung, A.K.W., Duewel, H.S., Honek, J.F., and Berghuis, A.M. (2001) 'Crystal structure of the lytic transglycosylase from bacteriophage lambda in complex with hexa-N-acetylchitohexaose’, Biochemistry, Vol. 40, pp. 5665-5673.

Perry, G. (2013) The Vanity of Small Differences, Hayward Publishing.

Pettersen, E.F., Goddard, T.D., Huang, C.C., Couch, G.S., Greenblatt, D.M., Meng, E.C. and Ferrin, T.E. (2004) 'UCSF Chimera - a visualization system for exploratory research and analysis', Journal of Computational Chemistry, Vol. 25, No. 13, pp. 1605-1612.

Phillips D.C. (1966) 'The three-dimensional structure of an enzyme molecule', Scientific American, Vol. 215, No. 5, pp. 78-90.

Physics arXiv Blog (2013) 'The art of chemistry - electronic visualisation', MIT Technology Review, 1 August 2013. URL: http://m.technologyreview.com/view/517506/the-art-of-chemistry-electronic-visualisation/ (accessed 10 May 2014).

Phipps, B. (2008). Beyond Measure: Conversations across art and science, Kettle's Yard, Cambridge, UK. URL: http://www.kettlesyard.co.uk/exhibitions/archive/beyond.html (accessed 10 May 2014). 
Portmann, S. and Lüthi, H.P. (2000) 'MOLEKEL: an interactive molecular graphics tool', CHIMIA International Journal for Chemistry, Vol. 54, No. 12, pp. 766-770.

Robinson, A. (2010a) Creative science versus artistic creation. In Sudden Genius? Oxford University Press, Oxford, Chapter 18, pp. 279-291.

Robinson, A. (2010b) Is there a creative personality? In Sudden Genius? Oxford University Press, Oxford, Chapter 19, pp. 292-303.

Schaftenaar, G. and Noordik, J.H. (2000) 'Molden: a pre- and post-processing program for molecular and electronic structures', Journal of Computer-Aided Molecular Design, Vol. 14, No. 2, pp. 123-134. DOI: 10.1023/A: 1008193805436

Smith, L.J., Bowen, A.M., Di Paolo, A., et al. (2013) 'The dynamics of lysozyme from bacteriophage lambda in solution probed by NMR and MD simulations', ChemBioChem, Vol. 14, No. 14, pp. 1780-1788.

Sula, C.A. (2013) 'Qualifying culture: four types of value in visualisation', Chapter 3, pp. 25-37, in Bowen, J.P., et al. (2013).

Trott, M. (1999) The Imaginary Made Real: The Art of Michael Trott, Wolfram Media, Graphica 1: The World of Mathematica Graphics.

University of Cambridge Museums (2014) Discoveries: Art, Science \& Exploration, Two Temple Place, London, pp. 1617.

van Doren, C. (1991) A History of Knowledge: Past, Present, and Future, Ballantine Books, New York.

Wallace, A.C., Laskowski, R.A., and Thornton, J.M. (1996) 'LIGPLOT: A program to generate schematic diagrams of protein-ligand interactions', Protein Eng., Vol. 8, pp. 127-134. PubMed id: 7630882

Weisberg, R.W. (2006) Creativity: Understanding Innovation in Problem Solving, Science, Invention, and the Arts, Wiley.

Wells A.F. (1956) The Third Dimension in Chemistry, Oxford University Press, Oxford.

Wikipedia (2014a) Chlorophyll a. URL: http://en.wikipedia.org/wiki/Chlorophyll_a (accessed 10 May 2014).

Wikipedia (2014b) List of molecular graphics systems. URL: http://en.wikipedia.org/wiki/List_of_molecular_graphics_systems (accessed 10 May 2014).

Wikipedia (2014c) Molecular graphics. URL: https://en.wikipedia.org/wiki/Molecular_graphics (accessed 10 May 2014).

Wolfram, S. (2002) A New Kind of Science, Wolfram Media. 